Draft VERSION JULY 12, 2018

Preprint typeset using IATEX style emulateapj v. 8/13/10

\title{
LOW-METALLICITY STAR FORMATION: PRESTELLAR COLLAPSE AND PROTOSTELLAR ACCRETION IN THE SPHERICAL SYMMETRY
}

\author{
Kazuyuki Omukai ${ }^{1,2}$, TAKashi Hosokawa ${ }^{1,2,3}$ AND NAOKI Yoshida 4 \\ Draft version July 12, 2018
}

\begin{abstract}
The collapse of dense cores with metallicities $0-1 Z_{\odot}$ is studied by hydrodynamical calculations coupled with detailed chemical and radiative processes. For this purpose, we construct a simple chemical network with non-equilibrium reactions among 15 chemical species, $\mathrm{H}^{+}, \mathrm{e}, \mathrm{H}, \mathrm{H}_{2}, \mathrm{D}^{+}, \mathrm{D}$, $\mathrm{HD}, \mathrm{C}^{+}, \mathrm{C}, \mathrm{CO}, \mathrm{CO}_{2}, \mathrm{O}, \mathrm{OH}, \mathrm{H}_{2} \mathrm{O}$, and $\mathrm{O}_{2}$, which reproduces the abundance of important molecular coolants by more detailed network very well. Starting from the initial density of $10^{4} \mathrm{~cm}^{-3}$, the evolution is followed until the formation of a hydrostatic protostar at the center $\sim 10^{21} \mathrm{~cm}^{-3}$. In a lowermetallicity gas cloud, the temperature during the collapse remains high owing to less efficient cooling. After the cloud core becomes optically thick to the thermal emission by dust, temperature evolution at the center converges to a single trajectory, except for cases with metallicity $\leq 10^{-6} Z_{\odot}$, where the temperature remains slightly higher than in higher metallicity cases even after becoming optically thick to thermal radiation by the $\mathrm{H}_{2}$ collision-induced emission. The protostellar masses at their formation are a few $10^{-3} M_{\odot}$, being slightly higher for cases with $\leq 10^{-6} Z_{\odot}$. Using the temperature evolution at the center as a function the density, we discuss the possibility of fragmentation during the dust-cooling phase. The critical metallicity for the fragmentation is $10^{-5} Z_{\odot}$ assuming moderate elongation of the cloud cores at the onset of this phase. From the density and velocity distributions at the time of protostar formation, we evaluate the mass accretion rate in the subsequent accretion phase. The accretion rate is larger than the Shu accretion rate for the inside-out collapse from an initially static cloud $\simeq c_{\mathrm{s}}^{3} / G$, where $c_{\mathrm{s}}$ is the sound speed in the prestellar gas, by about a factor of ten owing to more dynamical nature of the collapse. Using these accretion rates, we also calculate the evolution of the protostars under the assumption of stationary accretion flow. For $\geq 10^{-4} Z_{\odot}$, we succeed in following their evolution well after the arrival to the main-sequence phase. For lower metallicity cases, however, owing to too high accretion rates $\gtrsim$ a few $10^{-3} M_{\odot} / \mathrm{yr}$, the total luminosity, which consists of contribution from accretion and internal one, reaches the Eddington limit, thereby rendering the stationary accretion impossible for $\gtrsim 100 M_{\odot}$. Finally, we discuss possible suppression of fragmentation by heating of the ambient gas by protostellar radiation, which is considered important in the contemporary star formation. We argue that it is negligible for $<10^{-2} Z_{\odot}$.
\end{abstract}

Subject headings: stars: formation, stars: Population II

\section{INTRODUCTION}

The first stars in the universe are thought to be formed from a chemically pristine gas. Recent theoretical studies and cosmological simulations suggest that these stars are very massive, perhaps even greater than 100 solar masses (Bromm, Coppi \& Larson 1999; Abel, Bryan \& Norman 2002; Yoshida, Omukai \& Hernquist 2008; Ohkubo et al. 2009). Stars in the solar neighborhood are, on the other hand, typically of low-mass with the initial mass function peaking around $\sim 0.3 M_{\odot}$ (e.g., Kroupa 2002; Bastian et al. 2010). Although the characteristic mass of old halo stars - Population II stars- is not well known, a substantial fraction of them were formed with low masses and thus are still observed in the Galaxy. Clearly, there must have been a transition in the characteristic stellar mass from massive to low-mass ones.

Stars are formed under rather different conditions in

\footnotetext{
omukai@tap.scphys.kyoto-u.ac.jp

${ }^{1}$ Department of Physics, Kyoto University, Kyoto 606-8502, Japan

2 Division of Theoretical Astronomy, National Astronomical Observatory, Mitaka, Tokyo 181-8588, Japan

${ }^{3}$ Jet Propulsion Laboratory, California Institute of Technology, Pasadena CA 91109, USA

${ }_{4}^{4}$ IPMU, University of Tokyo, Kashiwa, Chiba 277-8583, Japan
}

the early universe and in the local universe. For example, metallicity of the interstellar gas, strength of a radiation field, existence of cosmic rays and a magnetic field, are all thought to be critical quantities that affect the process of star formation. Gas metallicity appears to be among the most important quantities that affects the thermal evolution of a prestellar gas and hence might determine the characteristic stellar (at least gas cloud) mass (e.g., Bromm et al. 1999; Omukai 2000; Schneider et al. 2002).

Observationally, no clear differences are found in the stellar initial mass function (IMF) for regions with different metallicities in the local universe (e.g., Kroupa 2002; Bastien, Covey, \& Meyer 2010). However, the difference of metallicity in the examined regions is only a factor of a few, and thus it remains unclear if the characteristic stellar mass can change drastically with much lower metallicity. Interestingly, there is an observational suggestion that very metal-poor stars in the halo had larger characteristic mass (Lucatello et al. 2005). The carbon-enhanced metal-poor stars are considered to be secondary, i.e., lower-mass, stars in binaries. The other star in the binary is typically an intermediate-mass star, whose mass is a few solar masses. The fraction of carbonenhanced stars increases with decreasing metallicity, in- 
dicating a higher fraction of intermediate-mass stars than in the solar neighborhood.

Observations show that, in the Galaxy, the shape of dense-core mass-function resembles that of the stellar IMF (e.g., Motte et al. 1998). This fact indicates that the characteristic stellar mass is originally imprinted through the formation process of the dense cores, e. g., fragmentation of star-forming gas. According to recent detailed numerical simulations, the frequency of fragmentation depends strongly on the temperature evolution of gas. Fragmentation occurs frequently as long as the effective ratio of specific heat $\gamma=\operatorname{dlog} p / \operatorname{dlog} \rho$, where $p$ is the pressure and $\rho$ is the density of the gas, is less than unity, i.e., the temperature decreases with increasing density during the collapse. With $\gamma>1$, on the other hand, the fragmentation hardly occurs (Li et al. 2003). The moment when $\gamma$ exceeds unity, i. e., at a temperature minimum, marks the end of the fragmentation epoch. The Jeans mass at this instant is imprinted as a characteristic fragmentation scale. Those numerical results can be interpreted by dynamical fragmentation theory of non-isothermal gas (Larson 1985), which predicts that the core mass-scale is set by fragmentation of filamentary clouds into round cores, which occurs when the isothermality breaks. In the first star formation, dense cores are indeed produced around the temperature minimum at $\sim 10^{4} \mathrm{~cm}^{-3}$, where the $\mathrm{H}_{2}$-cooling rate saturates owing to the collisional de-excitation. Also, for the present-day star formation, the Jeans mass at the dust-gas coupling, where the temperature becomes minimum, falls on the correct range for the characteristic stellar mass (Larson 1985; 2005, Whitworth et al. 1998).

Prestellar evolution of both present-day and primordial clouds has already been modelled in detail. In a primordial gas cloud, important processes are the chemistry and radiative processes of $\mathrm{H}_{2}$, which are simple enough to incorporate into numerical simulations in a direct, self-consistent manner (Omukai \& Nishi 1998; Abel, Bryan \& Norman 2002; Yoshida, Omukai \& Hernquist 2008). The situation is clearly different for the presentday star formation. Quite a large number of radiative and chemical processes are operating in a metal-enriched gas cloud, e.g., cooling by atomic and molecular line emission, heating by the dissipation of turbulence and by external radiation fields, in particular, in a diffuse medium. At first glance, this complexity makes it extremely hard to model the presente-day star formation process. In addition, once a central protostar turned on, the heating of ambient gas by its radiation becomes important (Krumholz 2006; Bate 2009). However, considering the fact that the cooling by dust grains dominates other radiative cooling processes after the formation of dense cores $\left(\gtrsim 10^{4} \mathrm{~cm}^{-3}\right)$ and before the protostar formation, a simplification is possible: the prestellar collapse of dense cores can be modelled by considering only the radiative transfer of dust thermal emission. This makes the chemistry and radiative process effectively decouple (Gaustad 1963; Hattori, Nakano, \& Hayashi 1969), and thus detailed calculations have been done since long ago (Larson 1969; Narita, Nakano, \& Hayashi 1970; Winkler \& Newman 1980; Masunaga \& Inutsuka 2000).

In Pop II star formation, the main difficulty lies in the coupling of chemical reactions and radiative cooling processes: one needs to solve chemical reactions to know the abundances of coolant species accurately. Miki \& Nakano (1975) and Yoshii \& Sabano (1980) studied the thermal evolution of collapsing low-metallicity clouds using one-zone models. Similarly, Bromm \& Loeb (2003) and Santoro \& Shull (2006) examined the role of finestructure line cooling in a low-metallicity cloud. These studies, however, do not follow chemical reactions but assume that metals in the gas phase remain in the atomic phase. Omukai (2000) and Omukai et al. (2005; hereafter O05) solved the detailed chemical reactions as well as radiative processes. They identified the importance of cooling by $\mathrm{CO}, \mathrm{OH}$ and $\mathrm{H}_{2} \mathrm{O}$ for metallicity $Z<10^{-1} Z_{\odot}$. There still remains overall uncertainties, however. The dynamics is affected by the thermal evolution via pressure force, so the treatment in the one-zone models has not yet been justified. Three-dimensional hydrodynamical calculations were also performed by several authors (Bromm et al. 1999; Smith \& Sigurdsson 2007; Smith et al. 2009). A slightly different approach is to use a prescribed effective equation-of-state in hydrodynamical calculations. In such studies, temperature evolution is given solely as a function of density, based on the result of one-zone models (Clark, Glover \& Klessen 2008; Tsuribe \& Omukai 2006, 2008, Machida et al. 2008; 2009). Jappsen et al. (2007; 2009a,b) included chemical reactions self-consistently in their hydrodynamical calculations, but followed only early evolution phase with density $\lesssim 10^{4} \mathrm{~cm}^{-3}$. None of these calculations provide a complete picture of prestellar gas collapse with hydrodynamics, chemistry, and radiative transfer.

In this paper, we study the collapse of star-forming dense cores with different metallicities using radiation hydrodynamics calculations including chemical reactions for important molecular coolants. We follow their evolution up to the formation of protostars at the center under the assumption of spherical symmetry. The structure of the cores at the moment of the protostar formation sets the initial conditions for the subsequent accretion phase of the protostar. In particular, it determines the protostellar accretion rate. Using the obtained accretion rates, we then calculate the evolution of protostars until they start hydrogen burning and arrive at the main sequence phase.

This paper is organized as follows. In Sec. 2, the numerical method is described. We present the prestellar evolution of dense cores in Sec. 3 and then the protostellar evolution in the accretion phase in Sec. 4. Sec. 5 is for discussion. Finally, we summarize the paper in Sec. 6. In Appendix A the chemical network solved is presented. Appendix B contains the fitting parameters for the $\mathrm{OH}$ and $\mathrm{CO}$ cooling functions.

\section{METHOD OF CALCULATION}

We calculate the gravitational collapse of the dense cloud cores with metallicities $Z=0-1 Z_{\odot}$ by solving the radiation transfer and chemical reactions as well as hydrodynamics under spherical symmetry. Starting from the initial density $\sim 10^{4} \mathrm{~cm}^{-3}$ at the center, we follow the evolution until shortly after the formation of a protostar at $\sim 10^{21} \mathrm{~cm}^{-3}$.

\subsection{Hydrodynamics}

We solve hydrodynamics using the Lagrangian difference method with the standard von Neumann-Richtmyer 
viscosity (see, e.g., Mihalas \& Weibel-Mihalas 1984). The results presented here are obtained with 200 grid points logarithmically spaced at the initial moment, with the innermost cell containing a mass of $10^{-6} M_{\odot}$. The outer boundary is fixed at its initial position. To see the numerical convergence of our results, we also carry out calculations for the same setting but with the number of the cells halved. We have confirmed that the results agree well.

\subsection{Cooling Processes}

We include the same cooling processes as in $\mathrm{O} 05$ with some updates of the coefficients. The total cooling rate $\Lambda$ consists of three processes, i.e., cooling via atomic and molecular line photons, cooling by continuum radiation, and the chemical cooling / heating:

$$
\Lambda=\Lambda_{\text {line }}+\Lambda_{\text {cont }}+\Lambda_{\text {chem }} .
$$

When the chemical heating exceeds the cooling, the last term $\Lambda_{\text {chem }}$ becomes negative.

\subsubsection{Line-emission Cooling}

The line-cooling processes included are those by finestructure transitions of CII, CI, and OI, and rotational transitions of $\mathrm{H}_{2}, \mathrm{HD}, \mathrm{CO}, \mathrm{OH}$, and $\mathrm{H}_{2} \mathrm{O}$. For $\mathrm{H}_{2}$, vibrational transitions are also considered. Atomic and molecular line photons are trapped in the core when the optical depth is large. This effect is taken into account by reducing the cooling rate by a emission line multiplying the escape probability $\beta_{\mathrm{L}}$;

$$
\beta_{\mathrm{L}}=\frac{1-e^{-\tau_{\mathrm{L}}}}{\tau_{\mathrm{L}}} e^{-\tau_{\mathrm{C}}},
$$

where $\tau_{\mathrm{L}}$ is the optical depth of the line and $\tau_{\mathrm{C}}$ is that of continuum. The optical depth is evaluated with the radially-integrated column density

$$
N_{\mathrm{X}}(r)=\int_{r} y(X) n_{\mathrm{H}} d r^{\prime},
$$

where $y(X)$ is the concentration of the emitting species $X$, and $n_{\mathrm{H}}$ is the number density of hydrogen nuclei. The above integral is over the radial interval where the velocity shift is within the line width; $\left|v\left(r^{\prime}\right)-v(r)\right|<$ $\left(v_{\mathrm{D}}\left(r^{\prime}\right)+v_{\mathrm{D}}(r)\right) / 2$. In Omukai \& Nishi (1998), transfer of $\mathrm{H}_{2}$ lines is solved not only by using correct averaging over solid angles but also by resolving frequency distributions within lines. By comparing the results with the same initial condition as Omukai \& Nishi (1998) but with our approximations for line radiation transfer, we have confirmed that the results agree very well.

The coefficient for $\mathrm{CO}, \mathrm{OH}$ and $\mathrm{H}_{2} \mathrm{O}$ line transitions are updated from $\mathrm{O} 05$. For $\mathrm{H}_{2} \mathrm{O}$, we use the cooling rate given by Neufeld \& Kaufman (1993) for $T>100 \mathrm{~K}$, and Neufeld, Lepp, \& Melnick (1995) for $T<100 \mathrm{~K}$. For $\mathrm{CO}$ and $\mathrm{OH}$, we calculated the cooling rates as in $\mathrm{O} 05$ by using the transition coefficients from Leiden Atomic and Molecular Database (Schoier et al. 2005) and fit the results by the same form of the formula as in Neufeld \& Kaufman (1993). The fitting parameters for the CO and $\mathrm{OH}$ cooling rates are summarized in Appendix A.

\subsubsection{Continuum-emission Cooling}

We solve transfer of continuum radiation by the variable Eddington factor method with grey approximation (Mihalas \& Weibel-Mihalas 1984). For a given mean intensity $J$, the dust temperature $T_{\mathrm{d}}$ is determined by the energy balance on the dust grains:

$$
\kappa_{\mathrm{d}} B\left(T_{\mathrm{d}}\right)=\kappa_{\mathrm{d}} J+\Gamma_{\text {coll }},
$$

where the Planck function $B(T)=\sigma_{\mathrm{B}} T^{4} / \pi, \kappa_{\mathrm{d}}$ is the Planck mean opacity of dust and the heating rate for the dust by collisions with gas particles $\Gamma_{\text {coll }}$ is given by (Hollenbach \& McKee 1979)

$$
\Gamma_{\text {coll }}=4.4 \times 10^{-6}(f / \rho)_{\mathrm{gr}} n_{\mathrm{H}}\left(\frac{T}{1000 \mathrm{~K}}\right)^{1 / 2}\left(T-T_{\mathrm{d}}\right),
$$

where $T$ is the gas temperature, and $(f / \rho)_{\text {gr }}$ is total volume of the dust per unit gas mass, which is taken from Pollack et al. (1994). For given gas and dust temperatures, the source function can be written as

$$
S=\frac{\kappa_{\mathrm{d}} B\left(T_{\mathrm{d}}\right)+\kappa_{\mathrm{g}} B(T)}{\kappa_{\mathrm{d}}+\kappa_{\mathrm{g}}},
$$

where $\kappa_{\mathrm{g}}$ is the gas continuum opacity. Once distribution of the source function $S$ is given, the mean intensity $J$ is found by carrying out standard ray-tracing method. We do iterations until the consistent distributions of $T_{\mathrm{d}}$ and $J$ are obtained. Once radiative transfer is solved, the cooling rates by dust and gas continuum emissions are given by

$$
\Lambda_{\mathrm{d}}=4 \pi \kappa_{\mathrm{d}}\left[B\left(T_{\mathrm{d}}\right)-J\right]
$$

and

$$
\Lambda_{\mathrm{g}}=4 \pi \kappa_{\mathrm{g}}[B(T)-J],
$$

respectively.

\subsubsection{Chemical Cooling and Heating}

The change in chemical binding energy works as heating or cooling of the gas. The heating and cooling associated the chemical reactions are treated in the same way as in Omukai (2000). We note here that important processes are heating/cooling by the $\mathrm{H}_{2}$ formation/dissociation, respectively.

\subsection{Chemical Processes}

We solve the reduced chemical network of O05, which correctly reproduces the evolution of major coolant species obtained by a more detailed network calculation. Our network includes 24 compounds of hydrogen, deuterium, carbon and oxygen. For the following 15 major species, non-equilibrium reactions are solved: $\mathrm{H}^{+}, \mathrm{e}, \mathrm{H}$, $\mathrm{H}_{2}, \mathrm{D}^{+}, \mathrm{D}, \mathrm{HD}, \mathrm{C}^{+}, \mathrm{C}, \mathrm{CO}, \mathrm{CO}_{2}, \mathrm{O}, \mathrm{OH}, \mathrm{H}_{2} \mathrm{O}, \mathrm{O}_{2}$ (see Table 1). The other 9 minor species, i.e., $\mathrm{H}^{-}, \mathrm{CH}, \mathrm{CH}_{2}$, $\mathrm{CO}^{+}, \mathrm{O}^{+}, \mathrm{OH}^{+}, \mathrm{H}_{2} \mathrm{O}^{+}, \mathrm{H}_{3} \mathrm{O}^{+}, \mathrm{O}_{2}^{+}$, which appear only as intermediaries of the reactions among the above major species, are set to be chemical equilibrium (see Appendix B for more details). By performing some experiments using the one-zone models, we confirmed that the evolution of the major species' abundances in this method agree very well with that in the full non-equilibrium treatment. 


\subsection{Initial Core Models}

Initial models for the hydrodynamical calculations are prepared using the early evolution in our one-zone model. The model is the same as that developed in O05, but some rate coefficients of radiative and chemical processes are updated to be the same as in the hydrodynamical models. Figure B1 shows the prestellar temperature evolution calculated by the one-zone model with different metallicities. The temperature and chemical abundances of the initial models are assumed to be radially constant, and their values are taken from the one-zone models at $10^{4} \mathrm{~cm}^{-3}$. For density and velocity distributions, we assume the critical Bonner-Ebert sphere at rest, which is the hydrostatic configuration on the verge of the collapse. To initiate gravitational collapse, we enhanced the density by twenty percent. The central number density is $1.2 \times 10^{4} \mathrm{~cm}^{-3}$ at the beginning of the hydrodynamical calculations. In addition to the case of primordial gas, $Z=0$, we study the cases every one dex in metallicity from $Z=10^{-6} Z_{\odot}([\mathrm{M} / \mathrm{H}]=-6)$ to $1 Z_{\odot}([\mathrm{M} / \mathrm{H}]=0)$. The total masses of the dense cores depend on the initial temperature at $1.2 \times 10^{4} \mathrm{~cm}^{-3}$ for the hydrodynamical calculations; $4.7([\mathrm{M} / \mathrm{H}]=0), 37(-1), 52(-2), 1.7 \times 10^{2}$ $(-3), 1.6 \times 10^{3}(-4), 2.3 \times 10^{3}(-5)$, and $2.4 \times 10^{3} M_{\odot}(-6$, and $Z=0)$. The outer boundary is fixed at its initial position.

\section{PRESTELLAR COLLAPSE}

Figure B2 presents the temperature evolution at the center of the prestellar cores as a function of the number density. The overall evolution is quite similar to that calculated by the one-zone model (Fig B1), justifying the one-zone treatment for the core evolution. There are, however, small disagreements, in particular, at high densities and for low metallicity cases. We defer detailed discussion on these differences to later in Sec. 3.4, but here describe which thermal processes control the temperature evolution at each metallicity. The contribution to the cooling and heating rates by individual processes are presented in Figure B3 for different metallicities. This should be compared with Figure 2 of O05, where similar plots for the one-zone models are presented. In Figure B5. the effective ratio of specific heat at the center $\gamma=d \ln p / d \ln \rho$, which tells the variation of pressure in response to the density variation, is shown for those cases. Note that $\gamma-1$ equals the gradient of the curve in Figure B2 for constant molecular weight. The effective ratio of specific heat is an important index to examine dynamical response of self-gravitating clouds to thermal evolution. For example, the clouds easily fragments as long as $\gamma<1$, while fragmentation is strongly prohibited for $\gamma>1(\mathrm{Li}$ et al. 2003). Another critical value is $\gamma=4 / 3$. If $\gamma$ exceeds this value, the dynamical collapse is halted as the pressure overcoming the gravity, and a hydrostatic object is formed.

\subsection{Thermal Evolution in the Metal-free Case}

In this section, we review thermal evolution of the cloud core of a metal-free gas. We then describe the effects of metallicity later in Sec. 3.2. We focus on deviations from the metal-free case. In the case of metallicity $[\mathrm{M} / \mathrm{H}]=-6$, metallicity effects are so small that the temperature evolution is almost identical to the metal-free one except a slight offset at highest densities $\left(\gtrsim 10^{20} \mathrm{~cm}^{-3}\right)$.

Let us summarize here the formation processes of $\mathrm{H}_{2}$, which play a crucial role in the thermal evolution. The evolution of $\mathrm{H}_{2}$ concentrations is presented in Figure B4, along with those in the cases with metals. Below $\sim 10^{8} \mathrm{~cm}^{-3}, \mathrm{H}_{2}$ is formed by the $\mathrm{H}^{-}$channel:

$$
\begin{aligned}
\mathrm{H}+\mathrm{e} & \rightarrow \mathrm{H}^{-}+\gamma \\
\mathrm{H}^{-}+\mathrm{H} & \rightarrow \mathrm{H}_{2}+\mathrm{e},
\end{aligned}
$$

catalyzed by a small amount of remaining electrons. With their recombination proceeding, the $\mathrm{H}^{-}$channel is quenched and the amount of formed $\mathrm{H}_{2}$ saturates at $\sim 10^{-3}$ (Fig. B4). After this plateau, the $\mathrm{H}_{2}$ abundance begins to increase again at $\sim 10^{8} \mathrm{~cm}^{-3}$ via the three-body $\mathrm{H}_{2}$ formation:

$$
2 \mathrm{H}+\mathrm{H} \rightarrow \mathrm{H}_{2}+\mathrm{H},
$$

and

$$
2 \mathrm{H}+\mathrm{H}_{2} \rightarrow \mathrm{H}_{2}+\mathrm{H}_{2} .
$$

All the hydrogen is converted to the molecular form via this channel by the density $\sim 10^{11} \mathrm{~cm}^{-3}$.

Next, let us see the cooling and heating processes (Fig. B3 a). Until very high density $\sim 10^{19-20} \mathrm{~cm}^{-3}$ is reached, cooling and heating are always almost balanced, so that the evolution is nearly isothermal with temperature differing only by a small factor whereas density increases by many orders of magnitudes. The effective ratio of specific heat $\gamma$ remains below $4 / 3$ but is above 1 in this period except for brief intervals around $10^{9} \mathrm{~cm}^{-3}$ and $10^{11} \mathrm{~cm}^{-3}$, where $\gamma$ falls slightly below unity (Fig. B5 a). The heating is owing to the compression, but for $10^{9}-10^{12} \mathrm{~cm}^{-3}$, where the $\mathrm{H}_{2}$ formation heating associated with the three-body reaction (eq. 11 below) dominates. For the cooling, the $\mathrm{H}_{2}$-line emission contributes most until $\sim 10^{13} \mathrm{~cm}^{-3}$, although some lines become optically thick at $\sim 10^{11} \mathrm{~cm}^{-3}$ and this suppresses the cooling rate gradually towards a higher density. The steep decline of the $\mathrm{H}_{2}$ line-cooling rate at $10^{16} \mathrm{~cm}^{-3}$ is due to the $\mathrm{H}_{2}$ collision-induced continuum absorption. Another molecular species in the metal-free gas, HD, is known to play an important role in cooling if a metal-free gas is once ionized (Uehara \& Inutsuka 2000; Nagakura \& Omukai 2006; Greif \& Bromm 2006; Yoshida, Omukai, \& Hernquist 2007; McGreer \& Bryan 2008). In our case, however, it only contributes comparably to $\mathrm{H}_{2}$ at a brief period at $\sim 10^{4} \mathrm{~cm}^{-3}$.

With gradual increase of temperature, the balance of chemical equilibrium between the $\mathrm{H}_{2}$ formation (eq. 11) and its inverse dissociation reactions shifts in favor of dissociation in the range $10^{13}-10^{15} \mathrm{~cm}^{-3}$. The cooling by this subtle dissociation is enough to compensate the compressional heating, because of the large binding energy of molecular hydrogen, $\chi=4.48 \mathrm{eV}$. At $\sim 10^{15} \mathrm{~cm}^{-3}$, $\mathrm{H}_{2}$ formation is somewhat resumed owing to the cooling by the $\mathrm{H}_{2}$ collision-induced continuum emission, which is a molecular analogue of free-free emission. At densities greater than $\sim 10^{16} \mathrm{~cm}^{-3}$, the cooling rate by this process declines rapidly when the central part of the core becomes optically thick to this same continuum. This time, with increase of temperature, the $\mathrm{H}_{2}$ begins to dissociate. The dissociation cooling balances with the compressional heating until $\sim 10^{20} \mathrm{~cm}^{-3}$, where the dissociation 
is almost completed. For higher densities, without efficient cooling process, the temperature increases almost adiabatically and $\gamma$ exceeds $4 / 3$. This marks the end of the prestellar collapse and the formation of a hydrostatic object, i.e. a protostar, at the center (see Sec. 3.3).

\subsection{Metallicity Effects on Thermal Evolution}

Addition of metals alters the thermal evolution of starforming cloud cores significantly in the following ways.

\subsubsection{Atomic and Molecular Metal-line Emission}

For a metal-free cloud core, the efficient radiative cooling is solely by $\mathrm{H}_{2}$, i.e. its rovibrational line emission and collision-induced emission, with some contribution from HD around $\sim 10^{4} \mathrm{~cm}^{-3}$. With metal enrichment, the cooling by line emission of metallic atoms ([CII], [CI], $[\mathrm{OI}])$ and molecules $\left(\mathrm{CO}, \mathrm{OH}, \mathrm{H}_{2} \mathrm{O}\right)$ affects the thermal evolution.

Oxygen-bearing molecules, $\mathrm{OH}$ and $\mathrm{H}_{2} \mathrm{O}$, act as efficient coolants. In the case of the metallicity $[\mathrm{M} / \mathrm{H}]=-5$, since the gas temperature remains high at $>200 \mathrm{~K}, \mathrm{OH}$ molecules formed at $\sim 10^{7} \mathrm{~cm}^{-3}$ are readily converted to $\mathrm{H}_{2} \mathrm{O}$. Cooling by $\mathrm{H}_{2} \mathrm{O}$ rotational transition exceeds $\mathrm{H}_{2}$ cooling in the range $10^{8}-10^{10} \mathrm{~cm}^{-3}$. With more metals of $[\mathrm{M} / \mathrm{H}]=-(3-4)$, the lower temperature conditions delay the conversion of $\mathrm{OH}$ to $\mathrm{H}_{2} \mathrm{O}$ molecules. The line emission by $\mathrm{OH}$ molecules dominates cooling at $10^{6}-10^{8} \mathrm{~cm}^{-3}$ before the $\mathrm{H}_{2}$-formation heating raises the temperature and promotes the conversion to $\mathrm{H}_{2} \mathrm{O}$ molecules. After that, $\mathrm{H}_{2} \mathrm{O}$ significantly contributes to cooling until $10^{10} \mathrm{~cm}^{-3}\left(10^{9} \mathrm{~cm}^{-3}\right)$ at $[\mathrm{M} / \mathrm{H}]=-4([\mathrm{M} / \mathrm{H}]=-3$, respectively), when the dust thermal emission becomes important. With higher metallicity, even lower temperature due to the $\mathrm{CO}$ cooling makes $\mathrm{OH}$ and $\mathrm{H}_{2} \mathrm{O}$ hard to be excited and their importance in cooling diminishes although, for $[\mathrm{M} / \mathrm{H}]=-2$, OH cooling is still important for $10^{6}-10^{7} \mathrm{~cm}^{-3}$. For $[\mathrm{M} / \mathrm{H}] \gtrsim-2$, the fine-structure line transitions of carbon and oxygen are important for cooling in a diffuse phase $\left(\lesssim 10^{3} \mathrm{~cm}^{-3}\right)$, which is below our initial densities and not shown in Figure B3. At these metallicities, CO becomes the dominant coolant before it is overtaken by dust thermal emission. For $[\mathrm{M} / \mathrm{H}]=0$, this shift happens $\sim 10^{3} \mathrm{~cm}^{-3}$ and the CO cooling does not appear in Figure B3.

\subsection{2. $\mathrm{H}_{2}$ Formation on Dust Grains}

In a metal-free gas, only a small amount of $\mathrm{H}_{2}$ with concentration $y\left(\mathrm{H}_{2}\right) \sim 10^{-3}$ is formed via the gas-phase $\mathrm{H}^{-}$reaction channel before the onset of the three-body $\mathrm{H}_{2}$ formation at $\sim 10^{8} \mathrm{~cm}^{-3}$. In a metal-enriched gas, dust grains are usually present as well; efficient $\mathrm{H}_{2}$ formation proceeds on the grain surface even at low densities. Figure $\mathrm{B} 4$ shows that, at metallicity $[\mathrm{M} / \mathrm{H}] \gtrsim-5$, the $\mathrm{H}_{2}$ concentration continues increasing owing to the formation reaction on the dust, unlike the metal-free case where there is a plateau at $\sim 10^{-3}$. For higher metallicities, i.e., more dust grains, the $\mathrm{H}_{2}$ formation is more efficient, and its concentration is higher at the same density before becoming fully molecular by the three-body reaction $\left(\lesssim 10^{10} \mathrm{~cm}^{-3}\right)$. In the case of $[\mathrm{M} / \mathrm{H}]=-5$, for example, about three times more $\mathrm{H}_{2}$ are formed by the beginning of three-body reaction $\left(10^{8-9} \mathrm{~cm}^{-3}\right)$ than in the metal-free case. This higher $\mathrm{H}_{2}$ abundance causes a slight deviation in temperature from the metal-free case $\lesssim 10^{7} \mathrm{~cm}^{-3}$ even before the small temperature decrease by the $\mathrm{H}_{2} \mathrm{O}$ cooling in the range $10^{7-10} \mathrm{~cm}^{-3}$ (Fig. B2). With higher metallicity of $[\mathrm{M} / \mathrm{H}]=-(3-4)$, further $\mathrm{H}_{2}$ enhancement makes the temperature fall below the threshold for $\mathrm{HD}$ formation at $\simeq 150 \mathrm{~K}$. The temperature decreases by HD line cooling until its rotational levels reach LTE at $\sim 10^{5} \mathrm{~cm}^{-3}$ (Fig. B2). With even higher metallicity $([\mathrm{M} / \mathrm{H}] \gtrsim-2)$, despite of higher $\mathrm{H}_{2}$ abundance, the line cooling by metalic atoms $(\mathrm{OI})$ and molecules $(\mathrm{CO}, \mathrm{OH})$ is more significant than that with $\mathrm{H}_{2}$ and $\mathrm{HD}$ molecules.

Except for the case of the solar metallicity $([\mathrm{M} / \mathrm{H}]=0)$, $\mathrm{H}_{2}$ formation has not yet been completed by the initial density of calculation at $10^{4} \mathrm{~cm}^{-3}$. Heating associated with $\mathrm{H}_{2}$ formation has large effects on the temperature evolution. In the cases with lowest metallicities $([\mathrm{M} / \mathrm{H}] \leq$ $-5)$, the heating by three-body reaction is dominant over the compressional heating in the range $10^{9}-10^{12} \mathrm{~cm}^{-3}$. For metallicity of $[\mathrm{M} / \mathrm{H}]=-4 . .-1$, because of the low temperature at the onset of rapid $\mathrm{H}_{2}$ formation, the chemical heating develops a remarkable bump in the temperature evolution, separating two temperature dips, i.e. one at lower density by line cooling and the other at higher density by dust cooling. In particular, this effect makes a large jump in temperature evolution at $10^{8} \mathrm{~cm}^{-3}$ for $[\mathrm{M} / \mathrm{H}]=-4 . .-3$.

It should be noted that the rapid $\mathrm{H}_{2}$ formation phase and thus the resultant temperature bump shift towards lower density with higher metallicity. Also, the local maximum value of the temperature decreases with increasing metallicity. This is because, for the $\mathrm{H}_{2}$ formation in lower density, a larger fraction of the chemical binding energy is radiated away before collisions with other gas particles make it available for heating, along with more efficient radiative cooling by metals.

\subsubsection{Thermal Emission of Dust Grains}

Thermal energy of gas is efficiently transferred via collisions to dust grains, which has a lower temperature. The excess energy deposited on the grain is readily radiated as thermal radiation. This works as an efficient cooling mechanism at high densities where the collisions between gas and dust are frequent enough (see panels in Fig. B3), and makes a temperature $\operatorname{dip}$ for $[\mathrm{M} / \mathrm{H}] \gtrsim-5$ (Fig. [B2). The frequent collisions equalize the temperature of gas and dust (so-called dust-gas coupling). However, before the density increases enough that the dust-gas coupling occurs, the dust is cooler than the gas by a large margin. Note that we assume there is no external background radiation. In this case, the cooling rate by dust thermal emission obeys $\Lambda_{\text {dust }}=\Gamma_{\text {coll }} \propto Z n_{\mathrm{H}} T^{3 / 2}$ (see eq. 5), where the $Z$ dependence comes from $(f / \rho)_{\mathrm{gr}}$. The compressional heating rate is proportional to $n_{\mathrm{H}}^{1 / 2} T$ for the collapse at a rate of the free-fall. With increasing density, thus, the dust cooling becomes more important relative to the compressional heating and finally catches up with it. At this point the gas temperature falls down until it reaches the dust temperature. Thereafter, the cooling rate by dust emission is expressed as $\Lambda_{\text {dust }} \propto Z T^{\beta+4}$ in the optically thin case, where the Planck mean opacity of dust $\kappa_{\mathrm{d}} \propto T^{\beta}$. With keeping thermal balance between the dust cooling and compressional heating, temperature slowly increases as $\propto n_{\mathrm{H}}^{1 / 2(\beta+3)} \simeq n_{\mathrm{H}}^{0.1}$, where $\beta \simeq 2$ is 
used in the last expression.

Because of the dependence of $\Lambda_{\text {dust }}$ on $Z$, the dust cooling becomes less efficient for the lower metallicity and then the onset of its efficient cooling is postponed until the higher density regime, where the temperature is also higher. With metallicity below a threshold value $([\mathrm{M} / \mathrm{H}]=-6 . .-5)$, the temperature reaches the dust evaporation value before the dust cooling becomes efficient. In the case of $[\mathrm{M} / \mathrm{H}]=-6$, for example, the dust completely sublimates at $\sim 1500 \mathrm{~K}\left(2 \times 10^{14} \mathrm{~cm}^{-3}\right)$ before its cooling reaching the level of compressional heating, although it does some contribution at $10^{13}-10^{14} \mathrm{~cm}^{-3}$. The temperature dip by dust cooling is absent in this case. With higher metallicities, the dust cooling reaches the compressional heating at some point and balances it thereafter until the dust thermal emission becomes optically thick and ineffective. After the core becomes optically thick to the dust absorption, the temperature increases almost adiabatically with increasing density as seen clearly for $[\mathrm{M} / \mathrm{H}] \geq-4$ in Figure B2. This phase is called the first adiabatic phase. We distinguish it from another adiabatic phase (second adiabatic phase) at a higher density $\left(\gtrsim 10^{21} \mathrm{~cm}^{-3}\right)$. Note that a cloud core with higher metallicity becomes optically thick to dust absorption at progressively lower density and temperature: for example, $10^{11} \mathrm{~cm}^{-3}$ for $Z_{\odot}$, and $10^{13} \mathrm{~cm}^{-3}$ for $10^{-4} Z_{\odot}$. Nevertheless, evolutionary paths of temperatures (Fig. B2) during and after the first adiabatic phase are similar each other for $[\mathrm{M} / \mathrm{H}] \gtrsim-5$ (see Omukai 2000 for further explanation). Finally, at $\simeq 1500 \mathrm{~K}$, the remaining dust species, i.e., silicates and iron, totally evaporate. The central part of the core is now free of dust and becomes transparent again although the surrounding matter is still optically thick. Because the surrounding gas has a lower temperature, the central part is still able to cool by continuum emission. This phase, however, lasts for a brief interval since the core soon becomes optically thick to the same continuum. The $\mathrm{H}_{2}$ dissociation begins at this moment and the temperature increases only gradually under the action of the dissociation cooling. After its completion at $\sim 10^{21} \mathrm{~cm}^{-3}$, the temperature increases adiabatically again. This is the second adiabatic phase. The evolution after the beginning of $\mathrm{H}_{2}$ dissociation is similar to the metal-free case, although the temperature is somewhat lower than that in the metal-free case.

\subsection{Dynamical Evolution}

We next describe dynamical evolution of the dense cores as a response to the thermal evolution seen above in Sec. 3.2. In Figure B6, the rate of enhancement of central density $\rho_{\mathrm{c}}$, which can be given by the inverse of the dynamical timescale, $t_{\mathrm{dyn}}^{-1}=\left(d \rho_{\mathrm{c}} / d t\right) / \rho_{\mathrm{c}}$ is shown relative to the inverse of the free-fall timescale $t_{\mathrm{ff}}^{-1}=$ $1 / \sqrt{\frac{3 \pi}{32 G \rho_{\mathrm{c}}}}$. By comparing Figures B5 and B6, we see an anti-correlation between the collapse rate $t_{\mathrm{ff}} / t_{\mathrm{dyn}}$ and $\gamma$ : where $\gamma$ is high (low), the collapse rate is low (high) owing to increasing (decreasing, respectively) pressure with collapse relative to the gravity. In particular, when $\gamma$ exceeds $4 / 3$, the dynamical collapse is halted and the collapse rate becomes very low with $t_{\mathrm{ff}} / t_{\mathrm{dyn}}<1$ (lower dotted horizontal lines in Fig. B6), indicating that the col- lapse is quasi-static, although the core contracts slowly by accretion of ambient matter.

In Figures B7, B13, we present radial distributions of density, temperature, velocity, and enclosed mass of the dense cores at different epochs until the formation of protostars for zero-metallicity, and metallicity of $[\mathrm{M} / \mathrm{H}]=-6$, $-5,-4,-3,-2,-1$, and 0 . Between successive plots, the increment of central density is $10^{2.5}$ times that of the previous one. The exception is the final phase shown, which corresponds to the state slightly after the end of the prestellar collapse. Similarity nature of the collapse is obvious in density distributions in Panels (a) of Figures B7. B13. This collapse closely resembles that of the LarsonPenston (LP) similarity solution (Larson 1969; Penston 1969), which describes the runaway collapse of isothermal clouds. In this solution, a flat-density central part of about a Jeans length collapses at a rate of $t_{\mathrm{ff}} / t_{\mathrm{dyn}}=3.0$. With increasing density, the Jeans mass and thus the matter in the central flat region decreases. Since the collapse timescale $t_{\mathrm{dyn}} \propto \rho_{\mathrm{c}}^{-1 / 2}$ becomes shorter and shorter with increasing density, a gas in low-density envelope is effectively leftover from the evolution of the central region during the collapse. The density distribution in the envelope has a power of index -2 with 4.4 times that of the singular isothermal sphere, while the infall velocity is constant at 3.3 times the sound speed in the LP solution. The same type of solutions for more general polytropic equation of state has been found by Yahil (1980). Figure B6 shows that the collapse rate at the center of our cores also becomes close to the value for the LP solution, which is $t_{\mathrm{ff}} / t_{\mathrm{dyn}}=3.0$ for the isothermal case and indicated by dotted horizontal line. Note that the value $t_{\mathrm{ff}} / t_{\mathrm{dyn}}$ higher than unity does not mean the collapse is faster than the free-fall, for which $t_{\mathrm{ff}} / t_{\mathrm{dyn}}=3 \pi / 2=4.7$ asymptotically. In some occasions, in particular for the cores in the dust-cooling phases, peak values of the collapse rate exceed the isothermal LP value of 3.0 slightly as $\gamma<1$ in those ranges. For $[\mathrm{M} / \mathrm{H}]=-4$ and -3 , the temperature jump by $\mathrm{H}_{2}$ formation heating at $\sim 10^{8} \mathrm{~cm}^{-3}$ is observed as a sharp peak in $\gamma$ in Figure B5. The slowdown of the collapse at this epoch results in a temporary small value of $t_{\mathrm{ff}} / t_{\mathrm{dyn}} \sim 0.5$ (Fig. B6] as downward spikes), which indicates that the collapse becomes quasistatic. Although the sudden slow-down of the collapse develops a shock around the heated core (see Figs. B9 and $\mathrm{B} 10 \mathrm{c}$ ), this heating phase lasts only for a brief period and then dynamical collapse is soon recovered. While $\mathrm{H}_{2}$ formation is in progress, small oscillations in the central density are imposed by the associated heating on the overall collapse. These oscillations are seen for $[\mathrm{M} / \mathrm{H}]=-$ 1 as notches around the peak of the bump in Figure B2. For other cases, they are not noticeable in Figure B2. but still observable in Figure B3 as notches in the compressional heating rates at $\mathrm{H}_{2}$ formation epoch. After the core becomes optically thick, temperature increases adiabatically and $\gamma$ exceeds $4 / 3$. The pressure overcomes the gravitational pull before long and a hydrostatic core forms. This hydrostatic core is often referred to the first protostellar core in literature as it appears before the genuine protostar, which is also called the second protostellar core. Although dynamical collapse is halted in this phase, the core continues contracting on average, but its oscillates acoustically many times, in response to the in- 
creasing mass of the hydrostatic core by accretion of the envelope material. Finally, at $\sim 10^{16} \mathrm{~cm}^{-3}$, dynamical collapse is resumed owing to the cooling by $\mathrm{H}_{2}$ dissociation. When the dissociation has almost completed, the temperature again begins to increase adiabatically. A hydrostatic core formed in this second adiabatic-phase grows eventually to a star and thus is called the protostar. The formation of the protostar marks the transition from the prestellar phase to the protostellar phase. Observationally, the protostars still in the early protostar phase are considered be the Class 0 objects, while the Class I objects are later but still in the protostar phase.

In the metal-free case, the temperature increases gradually at almost a constant rate from $10^{4} \mathrm{~cm}^{-3}$ to $10^{19} \mathrm{~cm}^{-3}$ without remarkable features. Consequently, the radial density profile does not deviate noticeably from that of the LP-type similarity solution until $\gtrsim 10^{20} \mathrm{~cm}^{-3}$, where the appearance of a hydrostatic core results in steepening of density gradient. For a polytropic gas whose equation of state is $P \propto \rho^{\gamma}$, the envelope density profile obeys $\rho \propto r^{-2 /(2-\gamma)}$ (Larson 1969; Yahil $1983)$. Since $\gamma$ takes the values of $1-1.2$ at $<10^{19} \mathrm{~cm}^{-3}$ (Fig. B5), the density gradient in the envelope is $\simeq-2.2$, which is slightly steeper than the value for the isothermal collapse, -2 . The three-body $\mathrm{H}_{2}$ formation makes a small dip in temperature evolution at $\sim 10^{10} \mathrm{~cm}^{-3}$ (Fig. B2), which is observed also in radial temperature distribution at $10^{2-3} \mathrm{AU}$ (Fig. B7 b). The formation of the protostar at $10^{21-22} \mathrm{~cm}^{-3}$ results in sudden halting of the inflow, which develops the accretion shock at the stellar surface $\sim 10^{-2} \mathrm{AU}$ (Fig. B7 c). Also, the protostar formation can be seen as steepening of density profile around the protostellar surface, rapid increase of temperature, and a plateau in the enclosed mass profile. The mass of the protostar at its birth is somewhat smaller than $\sim 10^{-2} M_{\odot}$. With metal enrichment as small as $[\mathrm{M} / \mathrm{H}]=-5$, the density, velocity, and mass profiles look similar to those in the metal-free case, although temperature profile deviates from the metal-free one at $\sim 10^{3} \mathrm{AU}$ due to $\mathrm{H}_{2} \mathrm{O}$ cooling and $\sim 1 \mathrm{AU}$ due to dust cooling (Fig. (B8). Owing to the difference in temperature evolution for $\gtrsim 10^{16} \mathrm{~cm}^{-3}$ (Fig. B2), the effective adiabatic index $\gamma$ exceeds the critical value $4 / 3$ at $10^{21} \mathrm{~cm}^{-3}$ for $[\mathrm{M} / \mathrm{H}]=-5$ and higher metallicity cases, while at $10^{20} \mathrm{~cm}^{-3}$ for $Z=0$ (see Fig. B5). Thus the formation of protostar is delayed to slightly higher density and, the mass and radius at protostar formation are somewhat smaller than the metal-free case.

For the case of $[\mathrm{M} / \mathrm{H}]=-4$, the heating by $\mathrm{H}_{2}$ formation makes a jump in temperature distribution at $\sim 10^{3} \mathrm{AU}$ (Fig. B9b). This causes slow-down of collapse and formation of a short-lived hydrostatic core, whose appearance can be seen as kinks in the density and mass profiles (Figs. B9a, d), and a shock in the velocity profile (Fig. B9.). A temperature dip by dust cooling is obvious at $\sim 10 \mathrm{AU}$. Almost simultaneously with the dust-gas coupling, the core becomes optically thick to the dust opacity. Subsequently, the temperature increases rapidly for $10^{14}-10^{16} \mathrm{~cm}^{-3}$, but $\gamma$ does not exceed $4 / 3$ (Fig. [B5]. The dynamical collapse continues although slowing down with $t_{\mathrm{ff}} / t_{\mathrm{dyn}}$ reaching unity at $10^{17} \mathrm{~cm}^{-3}$, which indicates the collapse is now quasi-static (Fig. B6). This slowdown is seen as a small spike at $\sim 0.1 \mathrm{AU}$ in the velocity profile (Fig. B9c). Even in a non-spherical collapse, a cloud becomes round by this heating and fragmentation in the later dust-cooling phase might be suppressed. If so, low-mass fragments and stars cannot be formed in the range $[\mathrm{M} / \mathrm{H}] \sim-4$. Tsuribe \& Omukai (2008) attributed the origin of apparent paucity of stars in the range $[\mathrm{M} / \mathrm{H}]$ $=-(4.8 .4 .0)$ in the halo to it. The evolution after the $\mathrm{H}_{2}$ dissociation $\gtrsim 10^{16} \mathrm{~cm}^{-3}$ is identical to the case of $[\mathrm{M} / \mathrm{H}]=-5$.

The case of $[\mathrm{M} / \mathrm{H}]=-3$ is qualitatively similar to $[\mathrm{M} / \mathrm{H}]=-4$ (Fig. B10). However, the $\mathrm{H}_{2}$ formation heating, as well as the temperature increase in the optically thick regime, have larger effects. Even before the rapid $\mathrm{H}_{2}$-formation heating at $10^{8} \mathrm{~cm}^{-3}$, slower heating has started and temperature increases gradually with $\gamma=1.1-1.2$, higher than $\simeq 1$ for $[\mathrm{M} / \mathrm{H}]=-4$ in the same regime. The collapse then is slower with $t_{\mathrm{ff}} / t_{\mathrm{dyn}} \simeq 1.5$ while it is $\simeq 2$ for $[\mathrm{M} / \mathrm{H}]=-4$. Thus the consequence of the rapid heating is almost complete halt of the collapse for a brief period, as observed in a deep trough in the velocity profile at 2000AU (Fig. B10 c). The central part becomes optically thick to the dust absorption at $10^{12} \mathrm{~cm}^{-3}$, and the adiabatic contraction phase continues until $10^{16-17} \mathrm{~cm}^{-3}$, where the dust sublimates and then the $\mathrm{H}_{2}$ dissociation follows. Note that since the adiabatic phase begins at lower density for higher metallicity and ceases at almost the same density, the adiabatic phase is more prolonged. As seen in Figure B6, the collapse becomes quasi-static for $\gtrsim 10^{15} \mathrm{~cm}^{-3}$ and a hydrostatic object - the first protostellar core - forms. Signs of the hydrostatic core formation, i.e., density steepening, temperature increase, velocity dip, and flattening of enclosed mass profile, are seen at $\sim 1$ AU in Figure B10.

In the case of $[\mathrm{M} / \mathrm{H}]=-2$ (Fig. B11), the $\mathrm{H}_{2}$ formation heating is gradual with $\gamma \simeq 1.3$ without a jump in temperature. Thus, no hydrostatic object forms in this phase. The hydrostatic objects (first hydrostatic core) forms at $10^{14} \mathrm{~cm}^{-4}$, after the central part becomes optically thick to dust absorption. The core starts collapsing dynamically again by dust evaporation and $\mathrm{H}_{2}$ dissociation. Finally, at $10^{22} \mathrm{~cm}^{-3}$, the second hydrostatic core, i.e. protostar, forms with the same condition as in other cases with $[\mathrm{M} / \mathrm{H}] \geq-5$.

For higher metallicities (Figs. B12 and B13), the qualitative nature of the evolution remains the same, but the onsets of both the efficient dust-cooling and adiabatic phases are shifted to lower densities. Because of the longer adiabatic phase, the appearance of the first hydrostatic cores become more conspicuous (at $\sim 1 \mathrm{AU}$ ). The radius of the first protostellar core becomes slightly larger with increasing metallicity. Note that for the solar metallicity $([\mathrm{M} / \mathrm{H}]=0)$ case, the initial $\left(\right.$ at $\left.10^{4} \mathrm{~cm}^{-3}\right)$ temperature is already low, the outer bump is missing in the temperature profile (Fig. B13b).

\subsection{Comparison with the One-zone Model}

Figures B1 and B2 show some differences between the hydrodynamical and one-zone models, despite the overall similarity. In general, temperatures tend to be higher in the hydrodynamical models than in the one-zone models. The differences are summarized as follows; (1) In the onezone model, all the evolutionary tracks converge for $\gtrsim$ $10^{16} \mathrm{~cm}^{-3}$, while in the hydrodynamical models, the two 
lowest metallicity cases $(Z=0$ and $[\mathrm{M} / \mathrm{H}]=-6)$ remain higher than in others. (2) At $[\mathrm{M} / \mathrm{H}]=-5$, the temperature dip by dust cooling around $10^{14} \mathrm{~cm}^{-3}$ in hydrodynamical model is not as obvious as in the one-zone model. (3) At $[\mathrm{M} / \mathrm{H}]=-3, \mathrm{H}_{2}$ formation heating results in a large temperature jump at $\sim 10^{8} \mathrm{~cm}^{-3}$ in the hydrodynamical model, while in the one-zone model it ends up with a gradual increase in temperature.

All these deviations can be attributed to the fact that the collapse rate of the core tends to be faster in the hydrodynamical model than that assumed in the onezone model. In the one-zone model, the dynamical timescale is assumed to be equal to the free-fall time, i.e., $t_{\mathrm{ff}} / t_{\mathrm{dyn}} \simeq 1$. In the hydrodynamical models, soon after the beginning of collapse from the rest at $10^{4} \mathrm{~cm}^{-3}$, the collapse rate exceeds $t_{\mathrm{ff}} / t_{\mathrm{dyn}}>1$, which indicates that the collapse is highly dynamical. It should be noted that the free-fall timescale is the time needed for the density of the homogeneous gas at rest to reach infinite if the pressure is negligible, and $t_{\mathrm{ff}} / t_{\mathrm{dyn}}$ higher than unity does not mean that the collapse is indeed faster than the free fall.

Observing convergence of temperature tracks in high densities in the one-zone model, Omukai (2000) demonstrated analytically that the cores that cool by continuum emission and collapse over a timescale proportional to the free-fall time have the same specific entropy when they become optically thick to the same continuum, regardless of their different metallicities. The same value of specific entropy assures the convergence of the evolutionary tracks after the cores become optically thick. In the discussion of Omukai (2000), however, the gas was assumed to be fully molecular when the core becomes optically thick to the continuum. In our hydrodynamical models, due to higher temperature than in the one-zone models for $\mathrm{Z}=0$ and $[\mathrm{M} / \mathrm{H}]=-6$, about $10 \%$ of hydrogen molecules are already dissociated when the core becomes optically thick to the $\mathrm{H}_{2}$ collision-induced absorption. The lower binding energy and thus higher specific entropy result in higher temperatures even after the core becomes optically thick.

\section{EVOLUTION OF PROTOSTARS IN THE ACCRETION PHASE}

In Sec. 3, we have seen the collapse of dense cores and formation of tiny protostars at the center. Although very small mass of $\lesssim 10^{-2} M_{\odot}$ at its birth, the protostar is surrounded by a large amount of gas in the envelope, which is a remnant of the parental core. The protostar thus grows in mass by orders of magnitude through accretion of these materials. This is the main accretion phase, corresponding to Class 0 and I phases, observationally.

When the protostar is not very massive $(\lesssim 10-$ $\left.100 M_{\odot}\right)$, stellar feedback to the accretion flow is not significant yet. Then the accretion rate is determined by the density and velocity structures in the envelope at the moment of protostar formation, which are shown in Figures B14 and B15, respectively. For density distributions, the number density multiplied by $r^{2}$ is presented to emphasize differences among the cases with different metallicities. As a general trend, both the density and velocity are higher for lower metallicity owing to higher prestellar temperature as is expected for the LP similarity solution, which has density 4.4 times the singular- isothermal-sphere value $\propto c_{\mathrm{s}}^{2} / r^{2} \propto T / r^{2}$ and infall velocity 3.3 times the sound speed $c_{\mathrm{S}} \propto T^{1 / 2}$ (Sec. 3.3). We evaluate the accretion rate as

$$
\dot{M}\left(M_{r}\right)=\left.4 \pi r^{2}(-v) \rho\right|_{M_{r}} .
$$

These values are shown in Figure B16 as a function of the enclosed mass $M_{r}$, which can be regarded as the protostellar mass $M_{*}$ at the time when the mass element of interest falls on the protostar. It should be noted that the accretion rates shown in Figure B16 are about ten times higher than the often cited values of $c_{s}^{3} / G$, which are valid for the accretion from the static singular isothermal sphere (Shu 1977), because of the dynamical nature of the collapse. For the LP solution, this factor is $\simeq 50$. Although the density distribution soon reaches that of LP solution, convergence of the velocity to the LP solution is slow and does not reach the value of LP solution during the actual collapse. This results in a smaller factor of $\sim 10$ enhancement instead of $\simeq 50$ from $c_{s}^{3} / G$ (Foster \& Chevalier 1983; Larson 2003)

In the case of metallicity $[\mathrm{M} / \mathrm{H}]=-3$, expansion, rather than infall, is observed at a small radial range owing to oscillation of the core at the sudden $\mathrm{H}_{2}$-formation heating. This results in the negative value of accretion rate by equation (13, thin line in Figure B16 at $\left.\sim 10 M_{\odot}\right)$ and followed by enhanced rate later. In this range, the rate does not give the correct accretion rate. In the following calculation of protostellar evolution, we use a rate extrapolated from lower protostellar mass until the accretion rate becomes the same as this value (dotted line in Figure B16] in this range.

With the accretion rate obtained above, we calculate the accretion evolution of protostars as in Hosokawa \& Omukai (2009a). In this method, originally developed by Stahler et al. (1980) and Stahler \& Palla (1986), a protostar model is constructed by solving ordinary stellar structure equations assuming the hydrostatic equilibrium, while the envelope is solved under the assumption of the stationary accretion with a given accretion rate (see Hosokawa \& Omukai 2009a for more details). Figure B17 shows the evolution of protostellar radius with increasing protostellar mass for different metallicities. The calculations are initiated with the protostar mass of $0.01 M_{\odot}$. For $[\mathrm{M} / \mathrm{H}] \leq-5$, the accretion rate is too high at the beginning and we cannot find convergent solutions for protostars by our numerical scheme. Then we start from a smaller rate and increases it with protostellar mass until the rate shown in Figure B16 is reached. For those cases, we show the protostellar radii only after the correct accretion rates are reached and transient behaviours disappeared. Since the accretion rates adopted are similar, this result resembles closely that in Figure 3 of Hosokawa \& Omukai (2009b), which shows the cases of $10 c_{\mathrm{s}}^{3} / G$, calculated by temperatures from the one-zone model. From Figure B17, we first notice that lower metallicity protostars have larger radii owing to higher accretion rates. Since a short accretion timescale does not allow accreted matter to cool enough near the stellar surface before embedded inside, a protostar with higher accretion rate has higher entropy content and thus larger radius. In all cases in Figure B17. the protostellar radius initially increases gradually with 
addition of newly accreted matter. When the protostar is small in mass, the opacity due to the free-free absorption is so high inside the star that the embedded matter has no time to release its heat (so-called the adiabatic accretion phase). The protostar with solar metallicity $([\mathrm{M} / \mathrm{H}]=0)$ has small entropy content and thus the entropy generation by deuterium burning influences the evolution, which is observed as an enhanced increase rate of radius at $\simeq 0.4 M_{\odot}$. In other cases, the deuterium burning has no large impact in their evolution. The gradual increase in radius in the adiabatic accretion phase is followed by abrupt swelling. With increase of protostellar mass, the temperature inside also increases and the opacity decreases as $\propto T^{-3.5}$. At some point, the decreased opacity allows the heat kept in the stellar interior to propagate outward by way of radiative diffusion. At this moment, the protostellar radius swells abruptly and thereafter decreases with throwing away the contained heat (so-called the Kelvin-Helmholtz (KH) contraction). Finally, when the heat generated by hydrogen burning compensates the radiative loss, the star reaches stable hydrogen burning phase (solid circles in Fig. B17) of the zero-age main sequence. If the accretion is not halted by other mechanism, for example, blow away of the envelope by radiative force onto the dusty envelope, quenching of mass reservoir in the envelope, etc, the protostar continues to grow in mass. The radius after the arrival of zero-age main sequence is larger for the higher metallicity star since more efficient hydrogen burning by the $\mathrm{CN}$ cycle halts the $\mathrm{KH}$ contraction earlier.

For cases with $[\mathrm{M} / \mathrm{H}] \leq-5$, the total luminosity, i.e. the sum of the contributions from the accretion $L_{\text {acc }}$ and interior one $L_{\text {int }}$, becomes close to the Eddington luminosity during the $\mathrm{KH}$ contraction because of the high accretion rate and decreasing radius; note that $L_{\text {acc }} / L_{\text {Edd }} \propto \dot{M} / R_{*}$. At this moment, the surface layer of the protostar is thrust outward and the protostellar radius begins to inflate violently. This marks the end of the stationary accretion. The accretion may stop completely if this eruption clears away all the matter in the envelope. Another possibility is that the mass accretion is regulated by this effect. If the accretion rate is temporarily decreased, the luminosity falls below the Eddington value. Thus the accretion may continue in a sporadic fashion. For other cases, we continue calculation until all the matter in the parental core is accreted. The arrival to the main-sequence occurs at higher protostellar mass for lower metallicity, e. g., at $50 M_{\odot}$ for $[\mathrm{M} / \mathrm{H}]=-$ 4 , while at $4 M_{\odot}$ for $[\mathrm{M} / \mathrm{H}]=0$. For $[\mathrm{M} / \mathrm{H}]=0$, due to a small amount of the mass reservoir $\left(4.7 M_{\odot}\right)$, the accretion ceases shortly after the arrival to the main-sequence.

\section{DISCUSSION}

\subsection{Radiative Transfer Effects}

In this paper, we have solved radiative transfer for the continuum by ray tracing method, while in the one-zone model we just reduced the cooling rate from the optically thin value $\Lambda_{\text {cont,thin }}$ by

$$
\Lambda_{\text {cont }}=\Lambda_{\text {cont,thin }} \min \left(1, \tau^{-2}\right),
$$

where $\tau$ is the optical depth of the continuum absorption. To see whether such a simple and local treatment works in hydrodynamical models, we also calculate the cases where the continuum cooling rate at radius $r$ is evaluated by using equation (14), where outward radial optical depth $\tau_{\mathrm{r}}$ is used for $\tau$. The evolution of central temperature is shown in Figure B18 (dashed lines), along with those with radiative transfer (solid lines) for comparison. The central evolution is reproduced very well by this simple treatment although the temperature is slightly lower near the opacity limit $\tau \simeq 1$ and higher when dust is evaporated at $\sim 10^{16-17} \mathrm{~cm}^{-3}$. The former is likely due to our artificial switching of the cooling rate by equation (14) from optically thin to thick cases. In the radiative transfer cases, this transition proceeds in a more gradual way since the cooling rate is somewhat reduced from the thin value for the optical depth $\lesssim 1$. For the $Z=0$ case, higher cooling rate near the opacity limit at $\sim 10^{16} \mathrm{~cm}^{-3}$ results in a slightly higher molecular fraction, which mitigates temperature increase in a later phase and delays the protostar formation (see Fig. B19). The higher temperature around the dust evaporation is explained as follows. In the dust evaporation phase, the central part is already devoid of dust and transparent, while the surrounding matter is still optically thick. Then the central part can deposit its heat to the cooler surroundings by the gas continuum radiation. In this case, the rate by equation (14) under-estimates the real value since the radial optical depth $\tau_{\mathrm{r}}$, which integrates all the opacity along the radial direction, is too large. This results in the higher temperature.

Our ray-tracing method works well to describe the core evolution accurately. However, it does not to reproduce radial temperature distribution very well. The radial temperature distribution at the protostar formation is presented in Figure B19 (dashed lines) for $Z=0$, $[\mathrm{M} / \mathrm{H}]=-4,-2$, and 0 . We see clearly deviations in temperature near the first protostellar core at 1-100AU. In the radiative transfer model, the temperature are higher owing to radiative heating by the inner warm gas. Higher entropy of this heated gas results in larger size of the first protostellar core in a later phase than evaluated by the central temperature (e.g., Tomida et al. 2010).

\subsection{Critical Metallicity for Dust-induced Fragmentation}

Armed with the temperature evolution obtained above, we now discuss the critical metallicity for fragmentation to produce low-mass objects. Tsuribe \& Omukai (2006) studied the fragmentation of dense cloud cores in the dust-cooling phase by three-dimensional numerical simulations. They followed the evolution of the core elongation $\mathcal{E}=a / b-1$, where $a(b)$ is the semi-major (minor) axis. In their simulation, a core with $\mathcal{E} \sim 1$ at the onset of the dust-cooling phase fragmented to multiple clumps when $\mathcal{E}$ reached a critical value $\mathcal{E} \simeq 30$, which corresponds to $\sim 3$ in the linear theory. For more details, we refer Tsuribe \& Omukai (2006). We apply this criterion to our hydrodynamic simulation as follows. First, we assume that the cores have modest elongations of about unity at the beginning of the dust-cooling phase (defined where $\gamma<1$ and cooling is dominated by the dust). We expect that the core fragments in this phase when the elongation extrapolated by the linear theory exceeds 3 . Figure B20 shows the elongation factor, which is defined as how many times the elongation has grown from its initial value for $[M / H]=-1 \ldots-5$. For $[M / H]=-6$, there is no clear dust-cooling phase, while for $[\mathrm{M} / \mathrm{H}]=0$, rapid 
dust-cooling phase with $\gamma<1$ has almost finished by the beginning of the hydrodynamical calculation at $10^{4} \mathrm{~cm}^{-3}$. For cases $[\mathrm{M} / \mathrm{H}]>-5$, the elongation factor well exceeds the critical value of 3 , while for the case with $[\mathrm{M} / \mathrm{H}]=-5$, it only marginally reaches 3 . For lower metallicities, it is clear that the elongation factor remains below 3 . We thus conclude that for cores initially elongated with $\mathcal{E} \sim 1$, the critical metallicity is $[\mathrm{M} / \mathrm{H}]=-5$ in our hydrodynamical models. Of course, whether fragmentation actually occurs depends on our assumption that the elongation is of order of unity at the beginning of the dust-cooling phase. In addition, in the discussion above, we used the temperature evolution derived by the calculation assuming spherical symmetry. When the core becomes significantly elongated, the collapse timescale as well as the cooling timescale will be different, which alters the temperature evolution. Note also that the dust-cooling rate depends on the uncertain dust-gas ratio and dust composition in the early universe (Schneider et al. 2006). For example, if the dust-gas ratio is lower than assumed here, the critical metallicity should be shifted upward with its inverse proportion. Also more refractory dust species, e.g., amorphous carbon rather than organic carbon, can survive in higher temperature condition of lower metallicity gas, thus enhancing the dust-cooling rate. Despite these uncertainties, the value of critical metallicity derived here provides an approximate estimate for further studies of low-metallicity star formation.

\subsection{Protostellar Heating to Prevent Fragmentation}

Stellar radiation heats up the surrounding material. For example, Vazquez-Semadeni et al. (2010) studied numerically the effect of the stellar ionization feedback from massive stars on the evolution of giant molecular clouds, and found that it controls their star formation efficiency. Since the Jeans mass gives the typical mass of fragments, this effect suppresses production of many tiny objects as a result of fragmentation. In the context of formation of present-day massive stars, Krumholtz (2006) pointed out that heating by accretion luminosity from a protostar alleviates fragmentation of the parental core, which facilitates monolithic collapse to a single massive object rather than fragmentation into a cluster of small ones. Krumholtz et al. (2007) confirmed this effect by raditation hydrodynamical calculation. Also, Bate (2010) found that radiative feedback from an accreting protostar suppresses fragmentation of the circumstellar disk by radiation hydrodynamical simulation. Such a raditive feedback could be potentially important also in a low-metallicity star forming core, owing to high accretion rate and thus luminosity of the central protostar and close separations between the fragments in the dustcooling phase. Here we examine whether this effect suppresses fragmentation in low-metallicity environments, as studied by Krumholz (2006) for the present-day star formation.

In the envelope of a protostar radiating at luminosity $L$, the dust is heated to a temperature $T_{\text {heat }}=$ $\left(L / 16 \pi \sigma r^{2}\right)^{1 / 4}$, which comes from the condition of radiative equilibrium. Note that this is just a measure for heating effect and is neither the actual dust nor gas temperatures: the true values of those temperatures are determined by taking account of the collisional coupling between the gas and dust. In the following, we compare the gas temperature $T_{\text {env }}$ in the envelope before the heating and $T_{\text {heat }}$, which is the maximum temperature by the heating effect in the region where $T_{\text {heat }}>T_{\text {env }}$. This dust temperature $T_{\text {heat }}$ is shown in Figure B21 for $\log \left(M_{*} / M_{\odot}\right)=-1.5,-0.5,0.5$ and 1.5. The innermost radii of the lines for $T_{\text {heat }}$ correspond to the positions of rarefaction waves. During the accretion phase, the inner matter falls onto the protostar earlier. The rarefaction front propagates outward with a sound speed and locates at $\sim 2 M_{*}$ in terms of the Lagrangian mass coordinate at the time of protostellar mass $M_{*}$ for the density distribution $\rho \propto r^{-2}$. The matter inside of the rarefaction front is falling at about a free-fall speed and probably is not affected by the protostellar heating anymore. In Figure B21. we see that $T_{\text {heat }}$ at a fixed radius increases with the protostellar mass due to increasing luminosity. However, the propagation of the rarefaction front is faster than increase of heating effect: the temperature at the rarefaction front decreases with increasing protostellar mass. Also, the effect of heating is less pronounced for lower-metallicity protostars. Below $[\mathrm{M} / \mathrm{H}] \leq-3, T_{\text {heat }}$ falls below $T_{\text {env }}$ at anytime outside the rarefaction front. Namely, the protostellar heating has no impact on the evolution because of the already high prestellar temperature. For $[\mathrm{M} / \mathrm{H}]=-2$ and -1 , the radiative heating may fill the temperature dip due to the dust cooling, but this phase lasts only for a short period. By $M_{*}=0.3 M_{\odot}$, this radiative heating effect becomes negligibly small. Only for $[\mathrm{M} / \mathrm{H}]=0$, the temperature due to the radiative heating is higher than the prestellar value throughout the evolution.

The tendency that radiative heating has more impact for higher metallicity (i.e., lower prestellar temperature) and for lower protostellar mass can be understood in the following way. When a protostar is not massive and still in the adiabatic phase, i.e., $M_{*} \lesssim 10 M_{\odot}$, its radius is approximated as (Stahler \& Palla 1986)

$$
R_{*}=48.1 R_{\odot}\left(\frac{M_{*}}{M_{\odot}}\right)^{0.27}\left(\frac{\dot{M}}{4.41 \times 10^{-3} M_{\odot} / \mathrm{yr}}\right)^{0.41} .
$$

We write the accretion rate as

$$
\begin{aligned}
\dot{M} & =\phi \frac{c_{\mathrm{s}}^{3}}{G} \\
& =1.63 \times 10^{-5} M_{\odot} / \mathrm{yr} \phi_{10} T_{10}^{3 / 2},
\end{aligned}
$$

by using a numerical factor $\phi(\sim 10$ in our case $)$, and $\phi_{10}=\phi / 10$ and $T_{10}=T / 10 \mathrm{~K}$. In the adiabatic phase, the protostellar luminosity is dominated by the contribution from the accretion luminosity:

$$
\begin{aligned}
L \simeq L_{\mathrm{acc}} & \simeq \frac{G M_{*} \dot{M}}{R_{*}} \\
& =1.01 \times 10^{2} L_{\odot} \phi_{10}^{0.59} T_{10}^{0.89} \mathrm{~m}_{*}^{0.73},
\end{aligned}
$$

where $m_{*}=M_{*} / M_{\odot}$. Thus, temperature of dust heated by the protostar is

$$
\begin{aligned}
T_{\text {heat }} & =\left(\frac{L_{\mathrm{acc}}}{16 \pi \sigma r^{2}}\right)^{1 / 4} \\
& =2.82 \times 10^{2} \mathrm{~K} \phi_{10}^{0.15} T_{10}^{0.22} m_{*}^{0.18} r_{10}^{-1 / 2},
\end{aligned}
$$


where $r_{10}=r / 10 \mathrm{AU}$. Suppose the location of rarefaction front $M_{\mathrm{rw}}=2 M_{*}$ and density distribution is a factor of $\alpha$ times that of the singular isothermal sphere $\rho=\alpha \rho_{\text {SIS }}$. For the Larson-Penston collapse $\alpha \simeq 4.4$, and we set it a representative value: $\alpha_{4.4}=\alpha / 4.4$. Using the radius of the rarefaction front

$$
\begin{aligned}
r_{\mathrm{rw}} & =\frac{G M_{*}}{\alpha c_{\mathrm{s}}^{2}} \\
& =5.59 \times 10^{3} \mathrm{AU} \alpha_{4.4}^{-1} T_{10}^{-1} m_{*} .
\end{aligned}
$$

We obtain the dust temperature due to protostellar heating at the rarefaction front

$$
T_{\text {heat }}\left(r_{\text {rw }}\right)=11.9 \mathrm{~K} \alpha_{4.4}^{0.5} \phi_{10}^{0.15} T_{10}^{0.72} m_{*}^{-0.32} .
$$

Therefore, the condition on the protostellar mass for $T_{\text {heat }}\left(r_{\text {rw }}\right)$ to exceed the prestellar temperature is given by

$$
M_{*}<1.7 M_{\odot} \alpha_{4.4}^{1.57} \phi_{10}^{0.46} T_{10}^{-0.88} .
$$

This demonstrates that the radiative heating effect is important only up to some mass given by equation (25), which decreases rapidly with increasing prestellar temperature, as has been discussed from Figure B21.

In the course of the accretion, circumstellar disks form owing to the angular momentum. The gravitational instability of rapidly accreting disks were investigated by Kratter et al. (2010) for the isothermal gas by way of global numerical experiments. They found that the central parameter for the stability of an accreting disk is $\xi \equiv \dot{M} /\left(c_{\mathrm{s}, \mathrm{d}}^{3} / G\right)=\phi\left(c_{\mathrm{s}} / c_{\mathrm{s}, \mathrm{d}}\right)^{3}$, which parameterized the accretion rate. Here $c_{\mathrm{s}, \mathrm{d}}$ is the sound speed in the disk and $c_{\mathrm{s}}$ is that in the parental core, i.e., before the matter falls on the disk. Rapidly accreting disks with $\xi \gtrsim 3$ become unstable and fragment into multiples or binaries, although the exact value for threshold depends on a rotational parameter of the parental cores, which stabilizes the disks somewhat. In our cases, since $\phi \sim 10$, the disks will become unstable and fragment without significant heating. Being closer to the star, cicumstellar disks can be more vulnerable to the radiative feedback than the matter outside the rarefaction wave, which has been discussed above. Although not discussed here, the radiative feedback onto the disk is interesting in this respect and awaits further investigations in future.

\section{SUMMARY}

We have studied star formation with different metallicities, $0-1 Z_{\odot}$, under the assumption of spherical symmetry. Until the birth of hydrostatic protostars, the gravitational collapse of a dense cloud core is calculated using chemo-radiation hydrodynamics. The subsequent protostellar accretion is followed by assuming the accretion flow is stationary while ordinary stellar structure equations are solved for the central protostar.

Our findings are summarized as follows:

(1) Prestellar temperature is higher in a lowermetallicity cloud core during optically thin stage, while it approaches a single density-temperature relation after the core becomes optically thick. Only in lowest metallicity $\left(\leq 10^{-6} Z_{\odot}\right)$ cases, the temperature remains slightly higher than other cases.

(2) Since the temperature evolution converges in cases with metallicity $\geq 10^{-5} Z_{\odot}$, physical conditions of pro- tostars at their formation are universal with a mass of $10^{-3} M_{\odot}$, a radius of $0.5 R_{\odot}$. The density and temperatures are $\sim 10^{22} \mathrm{~cm}^{-3}$ and $3 \times 10^{4} \mathrm{~K}$ respectively. In the cases with $\leq 10^{-6} Z_{\odot}$, owing to the slightly higher prestellar temperature, the protostar formation occurs about an order of magnitude earlier in density at $\sim 10^{21} \mathrm{~cm}^{-3}$, with slightly lower temperature $2 \times 10^{4} \mathrm{~K}$, and somewhat higher mass $2 \times 10^{-3} M_{\odot}$ and radius $1 R_{\odot}$.

(3) The prestellar collapse approaches the LarsonPenston self-similar solution, for which the velocity is 3.3 times the sound speed $c_{\mathrm{S}}$ and the density is 4.4 times the singular isothermal sphere value $\left(\propto c_{\mathrm{s}}^{2}\right)$. Reflecting the higher prestellar temperature, the velocity and the density in the envelope of a lower-metallicity dense core is higher at a given radius.

(4) The temperature evolution at the cloud center is roughly consistent with that found in our previous onezone models, although collapse is faster than assumed in the one-zone model, which results in a slightly higher temperature at the center. The faster collapse, in particular, affects the temperature in the dust-cooling phase. Since the fragmentation into low-mass objects occurs in this phase, the critical value of metallicity for inducing the fragmentation can be altered by this effect.

(5) The accretion rate after the birth of the protostar is higher in the lower-metallicity case because of the relation between accretion rate $\dot{M}$ and the prestellar sound speed $c_{\mathrm{s}}$ in similarity solutions, $\dot{M}=\phi c_{\mathrm{s}}^{3} / G$, where $\phi$ is a non-dimensional constant. For the isothermal LarsonPenston solution, which describes a highly dynamical collapse, $\phi=46.9$, while for the Shu solution, which is valid for accretion from a static isothermal sphere, $\phi=0.975$. In our cases, owing to modestly dynamical nature of the collapse, the accretion rates fall in between these two extremes and $\sim 10 c_{\mathrm{s}}^{3} / G$.

(6) The evolution of protostars under the accretion rates obtained from the prestellar collapse calculations is followed under the assumption of stationary accretion flows. The protostellar mass at its main sequence arrival is higher in the lower-metallicity case owing to the higher accretion rate: for example, it is $4 M_{\odot}$ for $1 Z_{\odot}$, while $50 M_{\odot}$ for $10^{-4} Z_{\odot}$. For $\leq 10^{-5} Z_{\odot}$, too high an accretion rate causes the luminosity to reach the Eddington limit and the accretion is almost halted at $80 M_{\odot}$. No stationary solution exists thereafter. The accretion may be halted temporarily. Once the accretion rate is reduced, however, the luminosity falls below the Eddington limit and thus the accretion would be resumed. The accretion may continue in such a non-stationary or a sporadic fashion.

(7) The critical metallicity for low-mass fragmentation is evaluated by applying the criterion by Tsuribe \& Omukai (2004) to the calculated temperature evolution at the center. With metallicity $Z \gtrsim 10^{-5} Z_{\odot}$, a moderately elongated cloud core is likely to fragment into low-mass objects during the dust-cooling phase.

(8) Radiation from a forming protostar heats up its ambient medium, thereby possibly preventing the fragmentation in the surrounding region. This protostellar heating effect is evaluated for different metallicity cases. For low-metallicities $\lesssim 10^{-2} Z_{\odot}$, the prestellar temperature is already high, and thus the heating effect is turned out to be insignificant. For higher metallicities, proto- 
stellar heating can be important especially in the early protostellar phase.

Effects such as rotation, magnetic fields, and turbulence, which are prevalent in the nearby star-forming clouds, have not been addressed in our spherical symmetric analysis. They would delay the contraction during the collapse phase and modify the accretion rate in the subsequent phase. In addition, rotation facilitates the fragmentation by forming a thin disk, while magnetic fields counteract it by extracting the angular momentum (Machida et al. 2008; Hennebelle \& Teyssier 2008). Those effects surely await future studies.

Overall, our calculations clarified the similarlity and differences in the formation of protostars with different metallicities. While some of the important issues, such as the critical metallicity for fragmentation, need further studies using full three-dimensional simulations, our results provide a foundation for such subsequent studies. Low-metallicity star formation is particularly important in the context of the formation of premeval galaxies. Most of the planned ground-based and space-borne telescopes are aimed at observing the first galaxies in which the gas metallicity is supposed to be very low. Theoretical studies such as those presented here will give an invaluable input for the future observations.

The present work is supported in part by the Grantsin-Aid by the Ministry of Education, Science and Culture of Japan (19047004, 2168407, 21244021:KO, 20674003:NY).

\section{REFERENCES}

Abel, T. G., Bryan, G., \& Norman, M. L., 2002, Sci, 295, 93

Bastian, N., Covey, K. R., \& Meyer, M. R., 2010, ARAA, arXiv:1001.2965

Bate, M. R., 2009, MNRAS, 392, 1363

Bate, M. R., 2010, MNRAS, 404, L79

Bromm, V., Coppi, P., \& Larson, R. B., 1999, ApJ, 527, L5

Bromm, V., Coppi, P., \& Larson, R. B., 2002, ApJ, 564, 23

Bromm, V., Ferrara, A., Coppi, P., Larson, R. B. 1999, MNRAS, 328, 969

Bromm, V. \& Loeb, A. 2003, Nature, 425, 812

Clark, P. C., Glover, S. C. O., \& Klessen, R. S. 2008, ApJ, 672, 757

Foster, P. N. \& Chevalier, R. A. 1993, ApJ, 416, 303

Greif, T. H., Johnson, J. L., Klessen, R. S., \& Bromm, V. 2008, MNRAS, 387, 1021

Hennebelle, P., \& Teyssier, R. 2008, A\&A, 477, 25

Hosokawa, T. \& Omukai, K. 2009a, ApJ, 691, 823

Hosokawa, T. \& Omukai, K. 2009b, ApJ, 703, 1810

Jappsen, A.-K. et al. 2005, A\&A, 435, 611

Jappsen, A.-K. et al. 2007, ApJ, 660, 1332

Jappsen, A.-K. et al. 2009a, ApJ, 694, 1161

Jappsen, A.-K. et al. 2009b, ApJ, 696, 1065

Kratter, K. M., Matzner, C. D.,, Krumholz, M. R., \& Klein, R. I., 2010, ApJ, 708, 1585

Kroupa, P. 2002, Science, 295, 82

Krumholz, M. R. 2006, ApJ, 641, L45

Krumholz, M. R., Klein, R. I., \& McKee, C. F. 2007, ApJ, 656, 959

Krumholz, M. R., Cunningham, A. J., Klein, R. I., \& McKee, C. F. 2010, preprint, arXiv:1001.0971

Larson, R. B. 1969, MNRAS 145, 271

Larson, R. B. 1985, MNRAS 214, 379

Larson, R. B. 2003, Reports on Progress in Physics, 66, 1651

Larson, R. B. 2005, MNRAS 359, 211

Li, Y., Klessen, R. S., \& Mac Low, M.-M. 2003, ApJ, 592, 975

Lucatello, S. et al. 2005, ApJ, 625, 833
Machida, M. N. 2008, ApJ, 682, L1

Machida, M. N., Tomisaka, K., Matsumoto, T., \& Inutsuka, S.-i. 2008, ApJ, 677, 327

Masunaga, H., \& Inutsuka, S. 2000, ApJ, 531, 350

Miki, S., \& Nakano, T. 1975, PASJ, 27, 147

Mihalas, D., \& Weibel-Mihalas, B. 1984, "Foundations of Radiation Hydrodynamics", Oxford Univ. Press

Neufeld, D. A., \& Kaufman, M. J. 1993, ApJ, 418, 263

Neufeld, D. A., Lepp, S., \& Melnick, G. J. 1995, ApJS, 100, 132

Omukai, K., \& Nishi, R. 1998, ApJ, 508, 141

Omukai, K., \& Palla, F. 2001, ApJ, 561, L55

Omukai, K., \& Palla, F. 2003, ApJ, 589, 677

Omukai, K. 2000, ApJ, 534, 809

Omukai, K., Tsuribe, T., Schneider, R., \& Ferrara, A. 2005, ApJ, 626,627

Palla, F. \& Stahler S. W. 1991, ApJ, 375, 288

Penston, M. V. 1969, MNRAS, 144, 425

Santoro, F. \& Shull, J. M. 2006, ApJ. 643, 26

Schneider, R., Ferrara, A., Natarajan, P., \& Omukai, K. 2002, ApJ, 571, 30

Schneider, R., Omukai, K., Inoue, A. K., \& Ferrara, A. 2006, MNRAS, 369, 1437

Schoier, F.L., van der Tak, F.F.S., van Dishoeck E.F., Black, J.H. 2005, A\&A432, 369

Shu, F. H. 1977, ApJ, 214, 488

Smith, B. D., \& Sigurdsson, S. 2007, ApJ, 661, L5

Smith, B. D. et al. 2009, ApJ, 691, 441

Tomida, K. et al. 2010 ApJ, 714, L58

Tsuribe, T. \& Omukai, K. 2006, ApJ, 642, L61

Tsuribe, T. \& Omukai, K. 2008, ApJ, 676, L45

Uehara, H., \& Inutsuka, S.-i. 2000, ApJ, 531, L91

Yahil, A. 1983, ApJ, 265, 1047

Yoshida, N., Omukai, K., \& Hernquist, L. 2007, ApJ, 667, L117

Yoshida, N., Omukai, K., \& Hernquist, L. 2008, Science, 645, 391

Yoshii, Y. \& Sabano, Y. 1980, PASJ, 32, 229 


\section{APPENDIX}

\section{A. REDUCED CHEMICAL NETWORK}

The set of reactions are presented in Table 1. The number of reactions and rate coefficients are the same as Table 1 of Omukai et al. (2005). This model includes 24 compounds of hydrogen, deuterium, carbon and oxygen, i.e., $\mathrm{H}^{+}$, e, $\mathrm{H}, \mathrm{H}_{2}, \mathrm{D}^{+}, \mathrm{D}, \mathrm{HD}, \mathrm{C}^{+}, \mathrm{C}, \mathrm{CO}, \mathrm{CO}_{2}, \mathrm{O}, \mathrm{OH}, \mathrm{H}_{2} \mathrm{O}, \mathrm{O}_{2} \mathrm{H}^{-}$. Non-equilibrium reactions are solved for the following 15 major species: $\mathrm{H}^{+}$, e, $\mathrm{H}, \mathrm{H}_{2}, \mathrm{D}^{+}, \mathrm{D}, \mathrm{HD}, \mathrm{C}^{+}, \mathrm{C}, \mathrm{CO}, \mathrm{CO}_{2}, \mathrm{O}, \mathrm{OH}, \mathrm{H}_{2} \mathrm{O}, \mathrm{O}_{2}$ (see Table 1). The other 9 species $\left(\mathrm{H}^{-}, \mathrm{CH}, \mathrm{CH}_{2}, \mathrm{CO}^{+}, \mathrm{O}^{+}, \mathrm{OH}^{+}, \mathrm{H}_{2} \mathrm{O}^{+}, \mathrm{H}_{3} \mathrm{O}^{+}, \mathrm{O}_{2}^{+}\right)$, which appears only as intermediaries of the reactions among the above major species, are set to be chemical equilibrium as follows:

$$
\begin{gathered}
\mathrm{H}^{-}: k_{2} n(H) n(e)=k_{3} n\left(H^{-}\right) n(H) \\
\mathrm{CH}:\left[k_{37} n(H)+k_{28} n\left(H_{2}\right)\right] n(C)+k_{22} n\left(C H_{2}\right) n(H)=n(C H)\left[k_{21} n(H)+k_{31} n\left(H_{2}\right)+k_{42} n(O)\right] \\
\mathrm{CH}_{2}: k_{62} n\left(H_{2}\right) n(C)+k_{31} n\left(H_{2}\right) n(C H)=k_{22} n\left(C H_{2}\right) n(H) \\
\mathrm{CO}^{+}: k_{48} n\left(C^{+}\right) n(O H)=k_{54} n\left(C O^{+}\right) n(H) \\
\mathrm{O}^{+}: k_{30} n\left(H^{+}\right) n(O)+k_{49} n\left(C^{+}\right) n\left(O_{2}\right)=\left[k_{50} n(H)+k_{51} n\left(H_{2}\right)\right] n\left(O^{+}\right) \\
\mathrm{OH}^{+}: k_{51} n\left(O^{+}\right) n\left(H_{2}\right)+k_{45} n\left(H^{+}\right) n(O H)=\left[k_{52} n\left(H_{2}\right)+k_{56} n(e)\right] n\left(O H^{+}\right) \\
\mathrm{H}_{2} \mathrm{O}^{+}: k_{52} n\left(O H^{+}\right) n\left(H_{2}\right)+k_{46} n\left(H^{+}\right) n\left(H_{2} O\right)=\left[k_{53} n\left(H_{2}\right)+\left(k_{57}+k_{58}\right) n(e)\right] n\left(H_{2} O^{+}\right) \\
\mathrm{H}_{3} \mathrm{O}^{+}: k_{53} n\left(H_{2} O^{+}\right) n\left(H_{2}\right)=\left(k_{59}+k_{60}\right) n(e) n\left(H_{3} O^{+}\right) \\
\mathrm{O}_{2}^{+}: k_{47} n\left(H^{+}\right) n\left(O_{2}\right)=k_{61} n(e) n\left(O_{2}^{+}\right) .
\end{gathered}
$$

From (A1)-A9 , the chemical equilibrium values are

$$
\begin{gathered}
y\left(H^{-}\right)=\frac{k_{2} y(e)}{k_{3}} \\
y(C H)=\frac{k_{37} y(C) y(H)+k_{28} y(C) y\left(H_{2}\right)+k_{62} y(C) y\left(H_{2}\right)}{k_{21} y(H)+k_{42} y(O)} \\
y\left(C H_{2}\right)=\frac{k_{62} y(C) y\left(H_{2}\right)+k_{31} y\left(H_{2}\right) y(C H)}{k_{22} y(H)} \\
y\left(C O^{+}\right)=\frac{k_{48} y\left(C^{+}\right) y(O H)}{k_{54} y(H)} \\
y\left(O^{+}\right)=\frac{k_{30} y\left(H^{+}\right) y(O)+k_{49} y\left(C^{+}\right) y\left(O_{2}\right)}{k_{50} y(H)+k_{51} y\left(H_{2}\right)} \\
y\left(O H^{+}\right)=\frac{k_{51} y\left(O^{+}\right) y\left(H_{2}\right)+k_{45} y\left(H^{+}\right) y(O H)}{k_{52} y\left(H_{2}\right)+k_{56} y(e)} \\
y\left(H_{2} O^{+}\right)=\frac{k_{52} y\left(O H^{+}\right) y\left(H_{2}\right)+k_{46} y\left(H^{+}\right) y\left(H_{2} O\right)}{k_{53} y\left(H_{2}\right)+\left(k_{58}+k_{57}\right) y(e)} \\
y\left(H_{3} O^{+}\right)=\frac{k_{53} y\left(H_{2} O^{+}\right) y\left(H_{2}\right)}{\left(k_{60}+k_{59}\right) y(e)} \\
k_{47} y\left(H^{+}\right) y\left(O_{2}\right) \\
k_{61} y(e)
\end{gathered}
$$

By using the one-zone models, we have compared the results by the chemical model above and those by fully nonequilibrium calculations with the same set of reactions and have found that the evolution of temperature, as well as abundances of the coolants abundances agree excellently. 


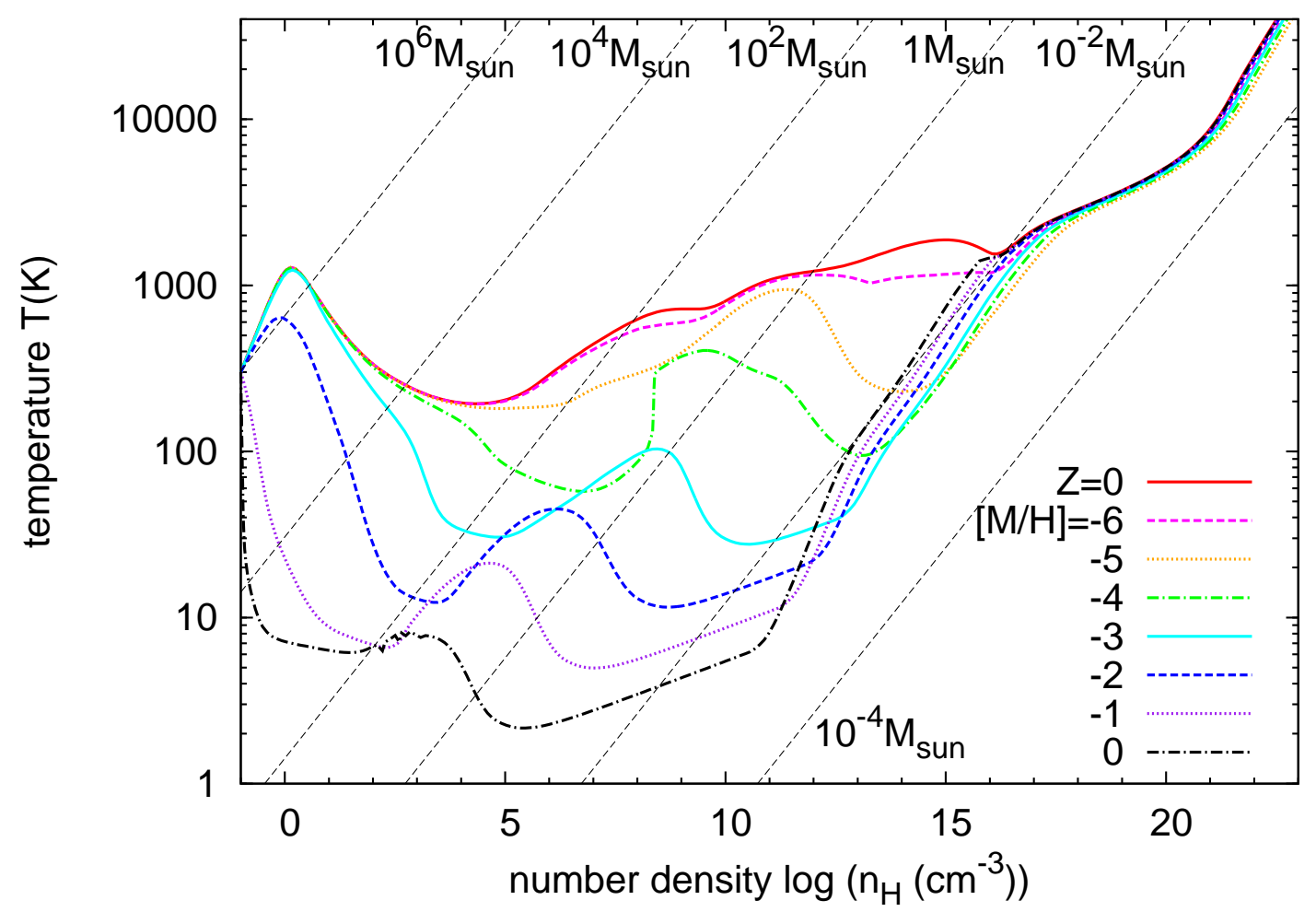

FIG. B1.- The evolution of temperatures in prestellar cloud cores with metallicities $Z / Z_{\odot}=0,10^{-6}, 10^{-5}, 10^{-4}, 10^{-3}, 10^{-2}, 10^{-1}$, and 1 , as functions of the number density, which is calculated by one-zone models. The dashed lines indicate the constant Jeans masses. For those above $10^{2} M_{\odot}$ (below $1 M_{\odot}$ ), the gas is assumed to be fully atomic (molecular) in drawing those lines.

\section{B. CO AND OH COOLING FUNCTIONS}

We compute the cooling rate of $\mathrm{CO}$ and $\mathrm{OH}$ rotational transitions by counting the level populations under the assumption of statistical equilibrium (see Omukai 2001 Appendix B). The level energies and transitional coefficients are provided by Leiden Atomic and Molecular Database (Schoier et al. 2005). The dependence of the results for molecule $\mathrm{M}$ on the three variables, the $\mathrm{H}_{2}$ number density $n\left(\mathrm{H}_{2}\right)$, column density parameter $\tilde{N}(\mathrm{M})$ and temperature $T$ is fitted by the following four-parameter expression according to Neufeld \& Kaufman (1993):

$$
\frac{1}{L_{\mathrm{M}}}=\frac{1}{L_{0}}+\frac{n\left(\mathrm{H}_{2}\right)}{\mathcal{L}_{\mathrm{LTE}}}+\frac{1}{L_{0}}\left[\frac{n\left(\mathrm{H}_{2}\right)}{n_{1 / 2}}\right]^{\alpha}\left(1-\frac{n_{1 / 2} L_{0}}{\mathcal{L}_{\mathrm{LTE}}}\right) .
$$

The column density parameter is defined as $\tilde{N}(M)=N(M) / \sqrt{\frac{2 k T}{m(\mathrm{M})}}\left(\mathrm{cm}^{-2} / \mathrm{km} \mathrm{s}^{-1}\right)$, where $N(\mathrm{M})$ is the column density, and $m(\mathrm{M})$ is the mass of molecule M. The fitting parameters $L_{0}$, which is a function of $T$ alone, and $\mathcal{L}_{\mathrm{LTE}}$, $n_{1 / 2}$, and $\alpha$, which are functions of both $\tilde{N}(M)$ and $T$, are presented in Table B2 for CO and in Table B3 for OH. 


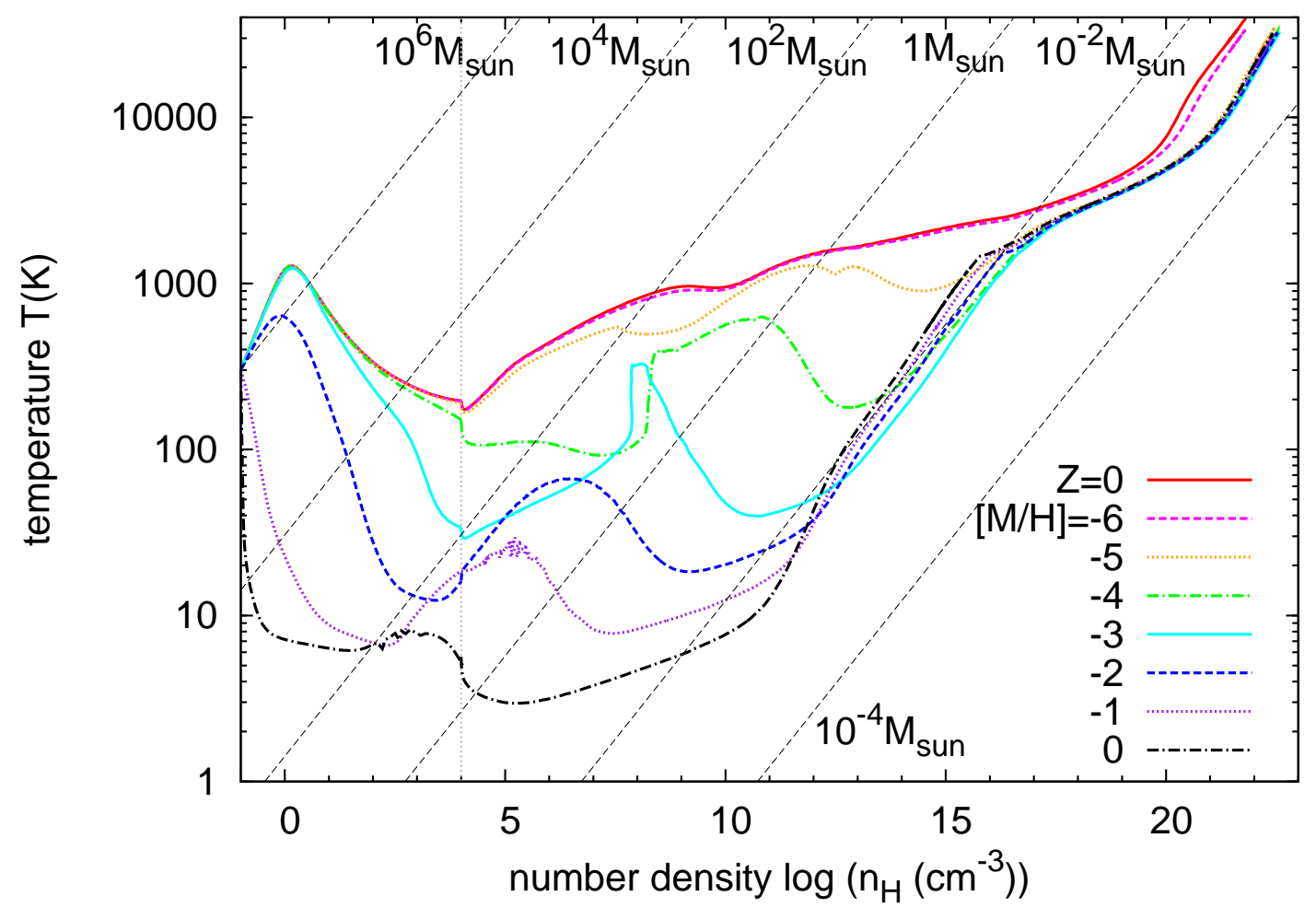

FIG. B2.- The evolution of temperatures at the center of cloud cores during the prestellar collapse for various metallicities. This is calculated by one-zone model until $10^{4} \mathrm{~cm}^{-3}$ (dotted vertical line) and by hydrodynamical models for the higher density. The constant Jeans masses are indicated by the dashed lines. 

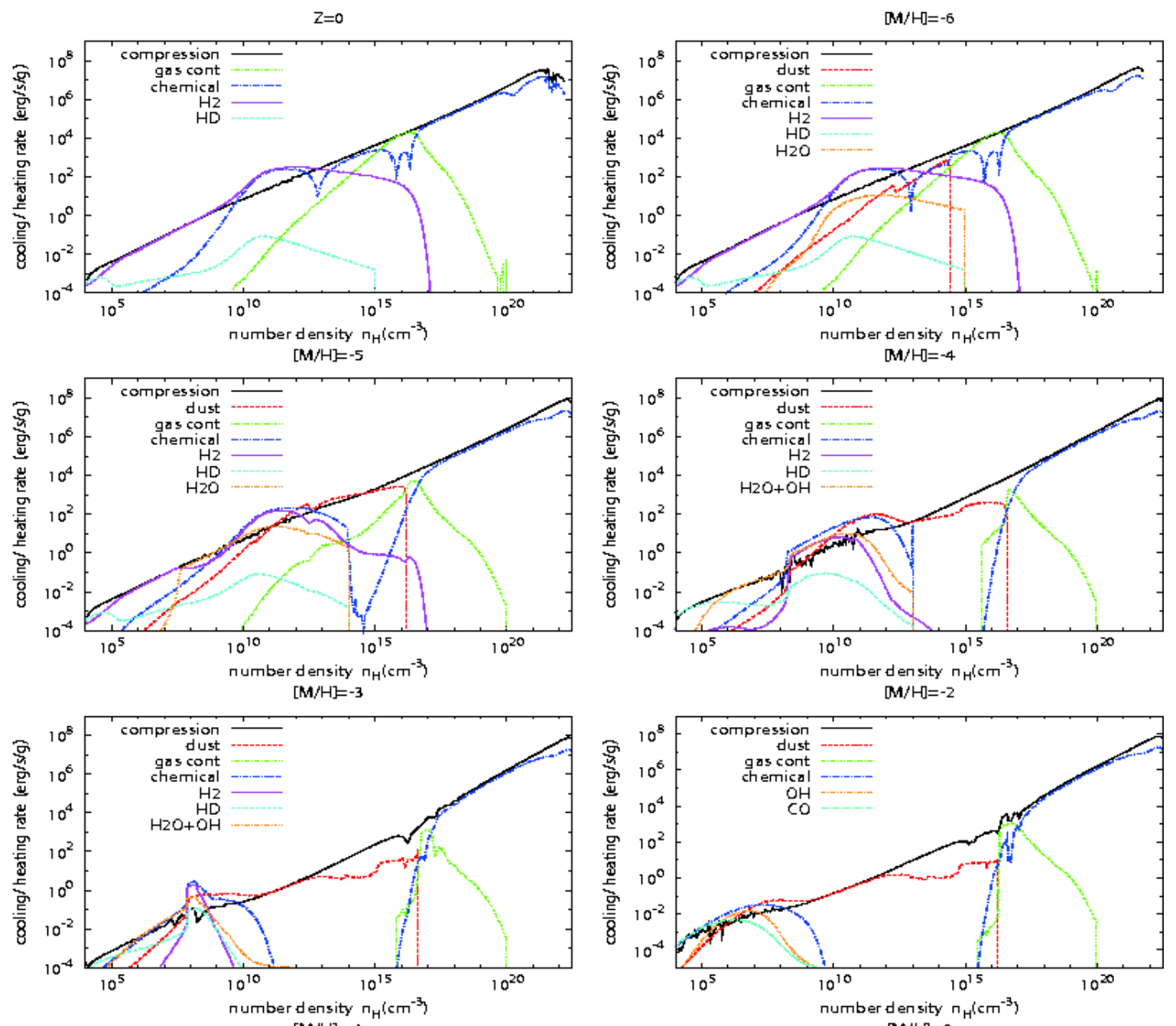

$[\mathrm{M} / \mathrm{H}]=-1$
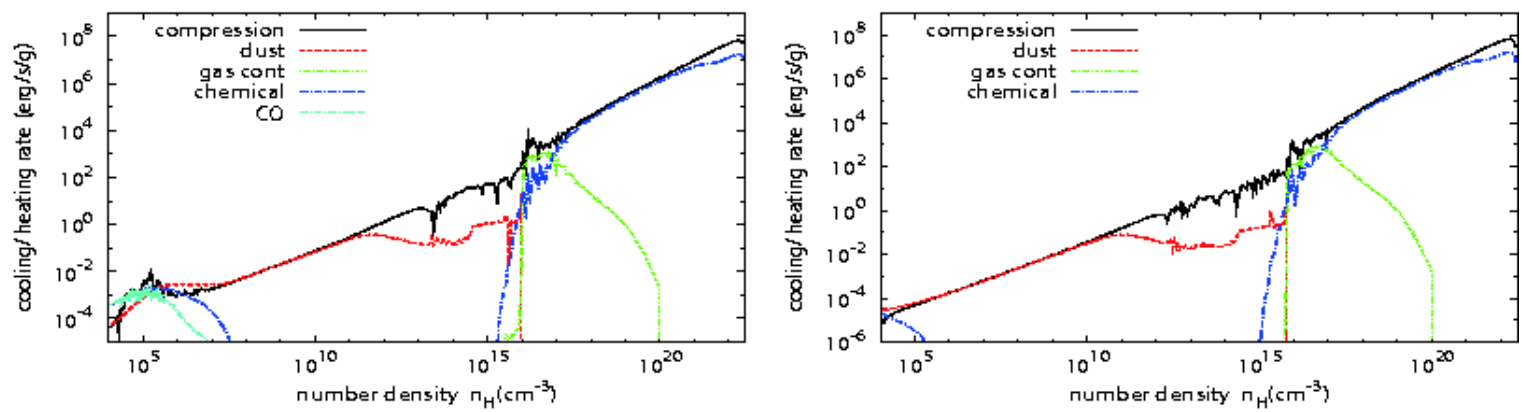

FIG. B3.- The cooling and heating rates per unit mass by individual processes at the centers of the cloud cores as functions of number density. To avoid violent oscillatory behaviors and keep the compressional heating rate positive during the adiabatic phases, we plot them at every time when the density exceeds 1.1 times the previous values. 


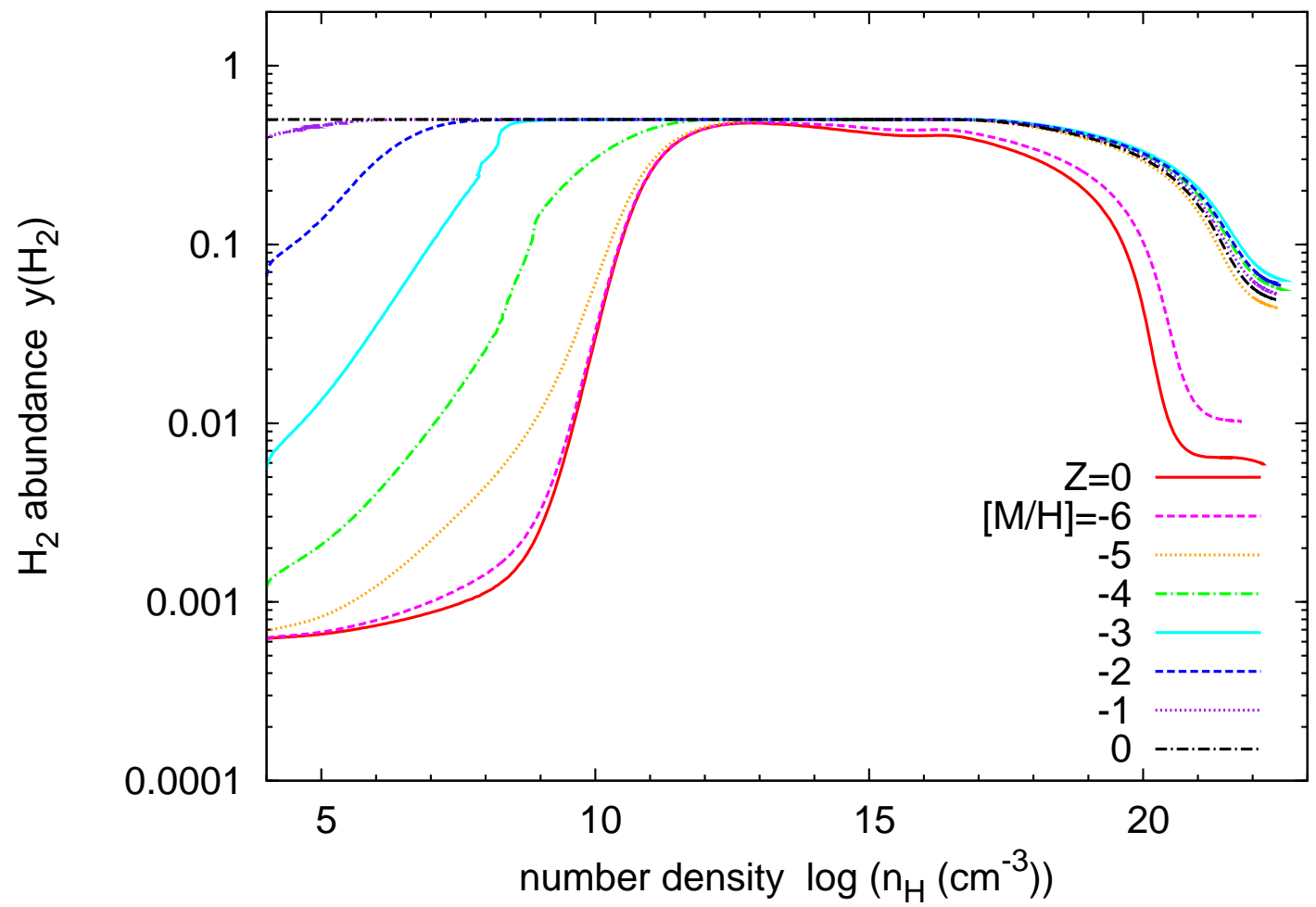

FIG. B4.- The evolution of the $\mathrm{H}_{2}$ concentrations $y\left(\mathrm{H}_{2}\right)$ at the centers of the cores. The state of $y\left(\mathrm{H}_{2}\right)=1 / 2$ corresponds to the fully molecular gas. 

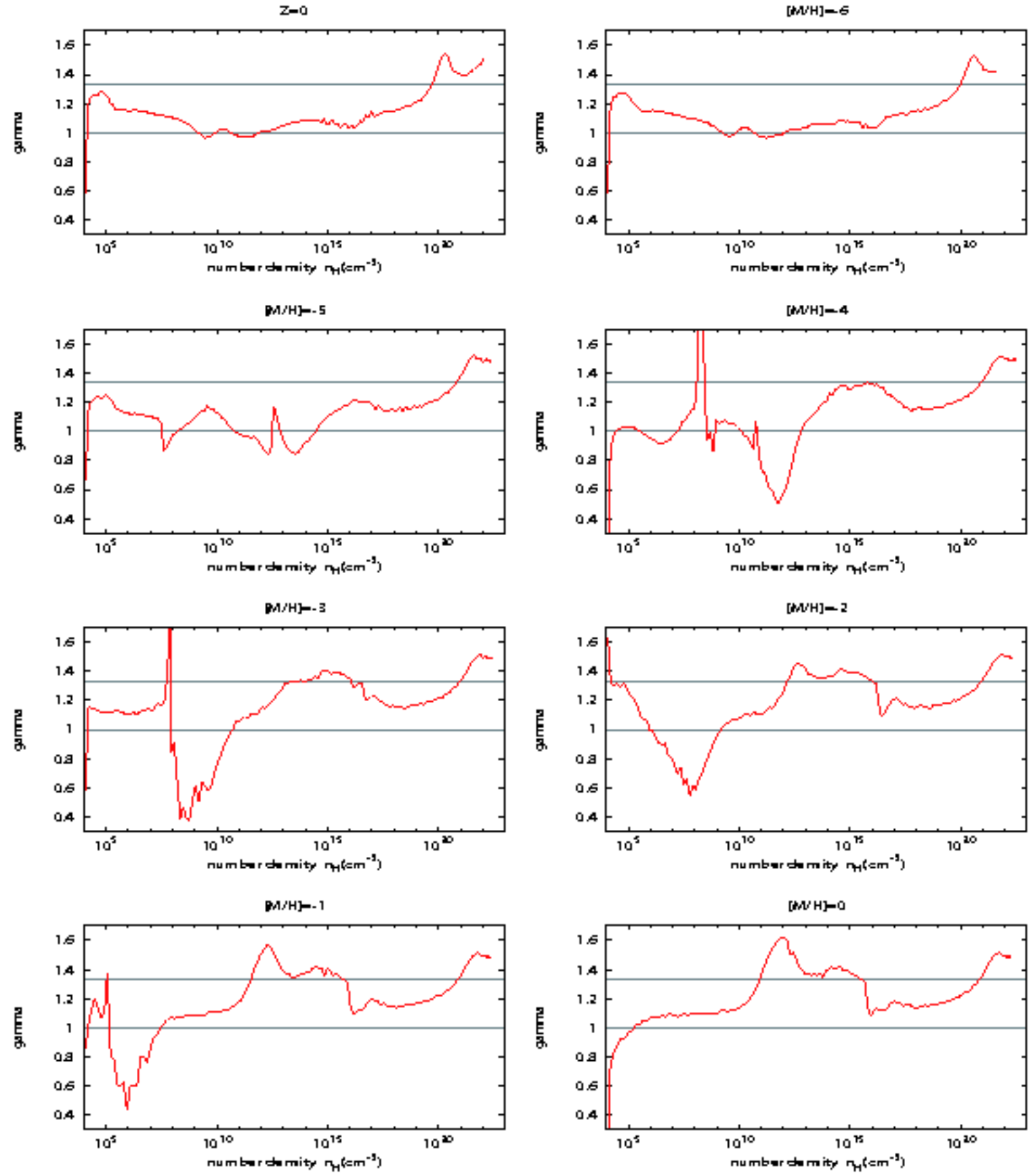

FIG. B5.- The effective ratio of specific heat $\gamma=\operatorname{d} \log p / \operatorname{dlog} \rho$ at the center during the prestellar collapse for different metallicities as a function of number density. The horizontal lines show the critical values for fragmentation (1) and for hydrostatic core formation (4/3), respectively. 

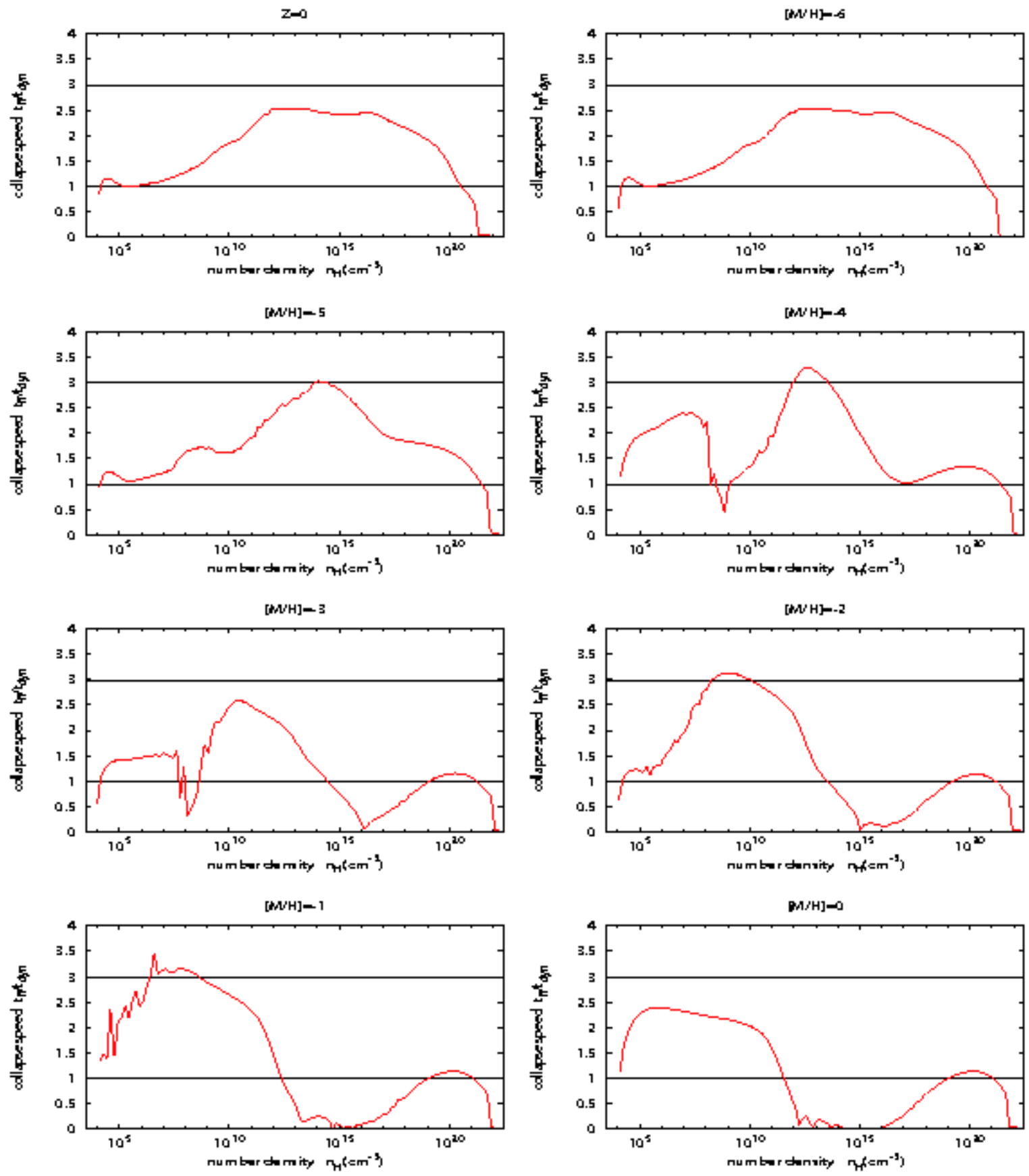

FIG. B6. - The collapse rate $t_{\mathrm{dyn}}^{-1}=\dot{\rho} / \rho$ at the center, normalized by the free-fall rate $t_{\mathrm{ff}}^{-1}$. The dashed horizontal curves indicate the value for the isothermal Larson-Penston similarity solution (3.0) and the threshold for quasi-static collapse (1.0). 

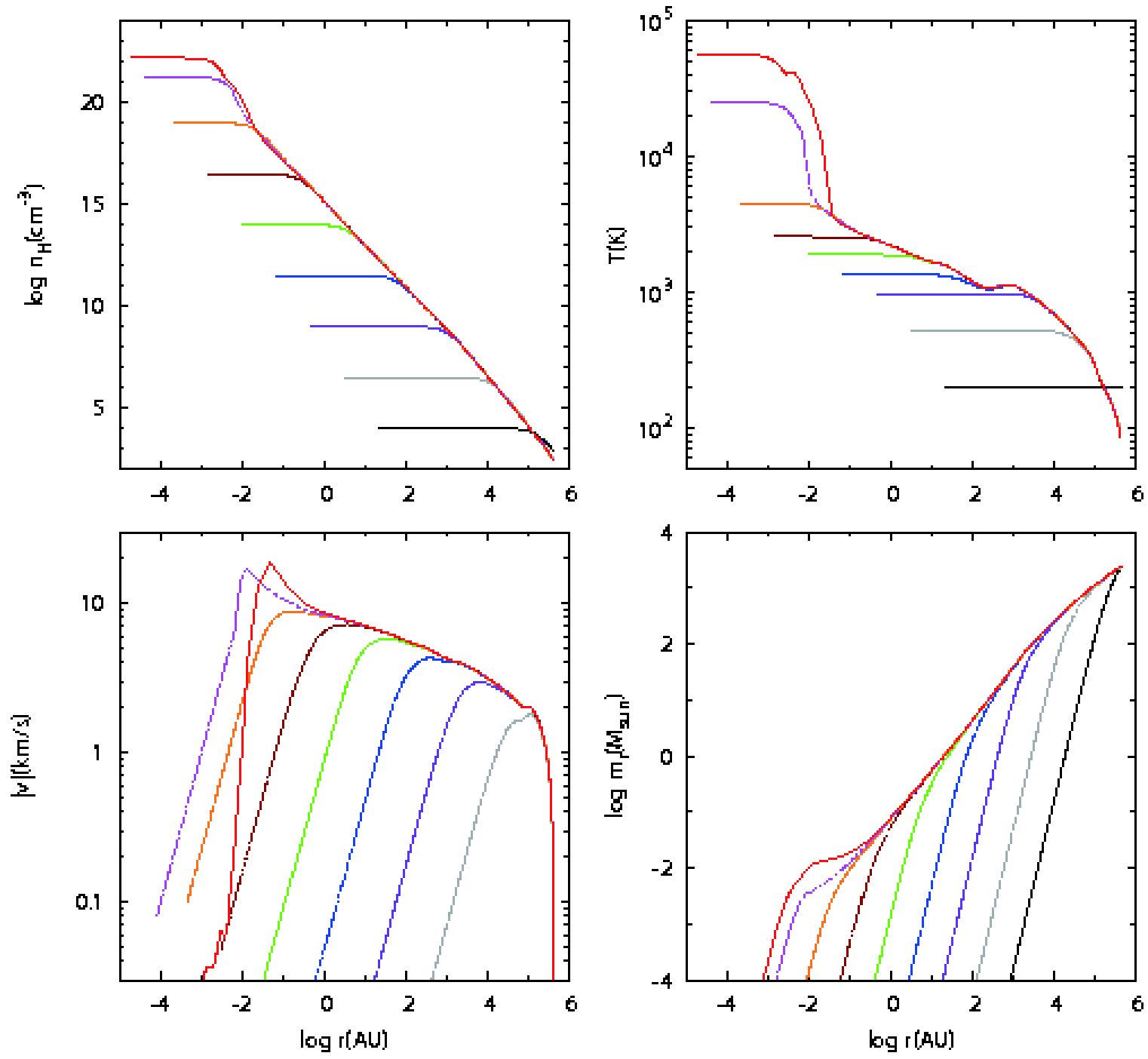

FIG. B7.- The radial distributions of (a) density (b) temperature (c) velocity and (d) enclosed mass at different epochs during the prestellar collapse for the cloud core with metallicity $\mathrm{Z}=0$. The first curves shown correspond to the initial state of the calculation. They are plotted when the central density is enhanced by a factor of $10^{2.5}$ from the previous one except the last state shown, which corresponds to the time somewhat after the formation of a protostar. 

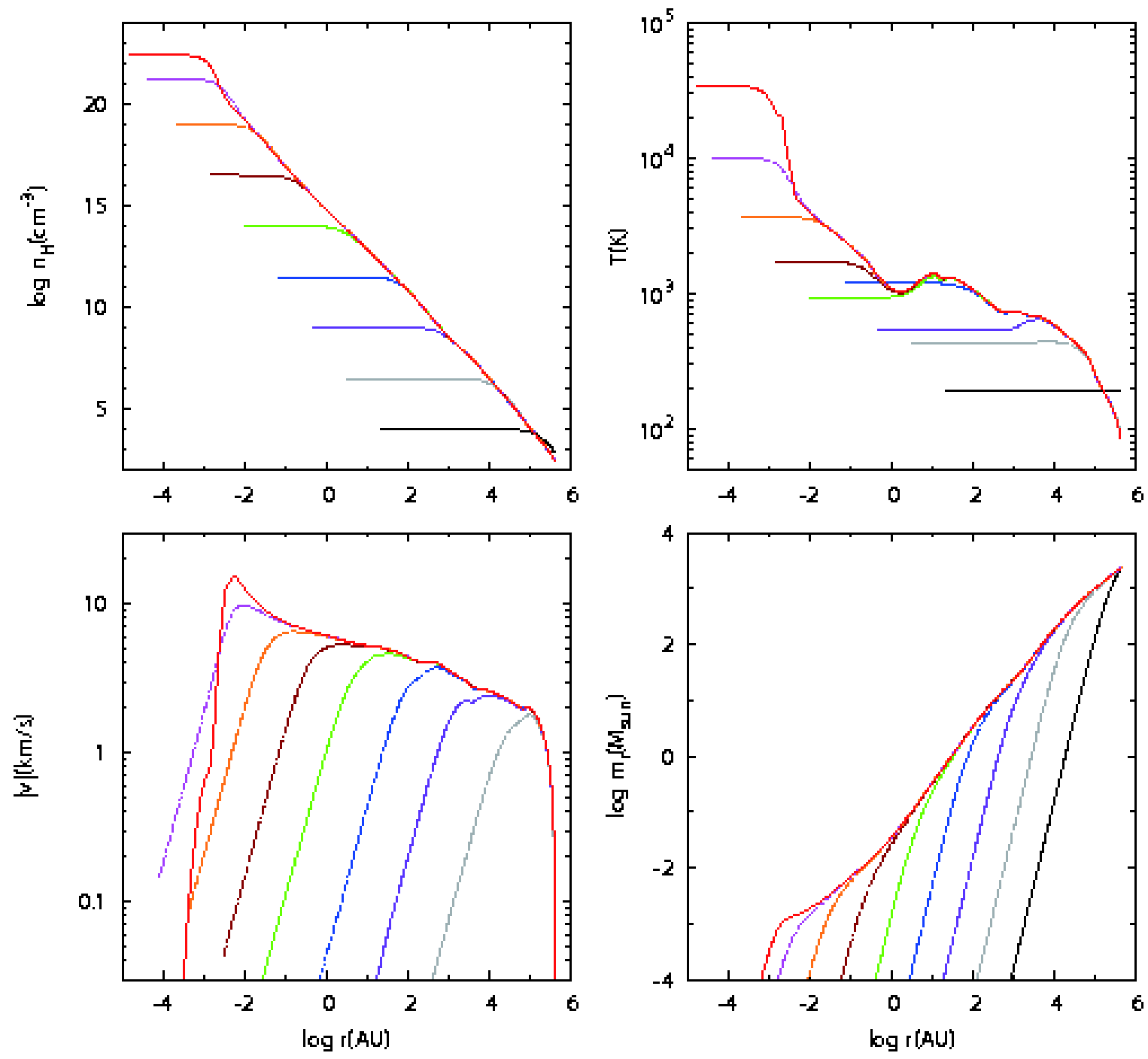

FIG. B8. - The same as Figure B7 but for metallicity $[\mathrm{M} / \mathrm{H}]=-5$ 

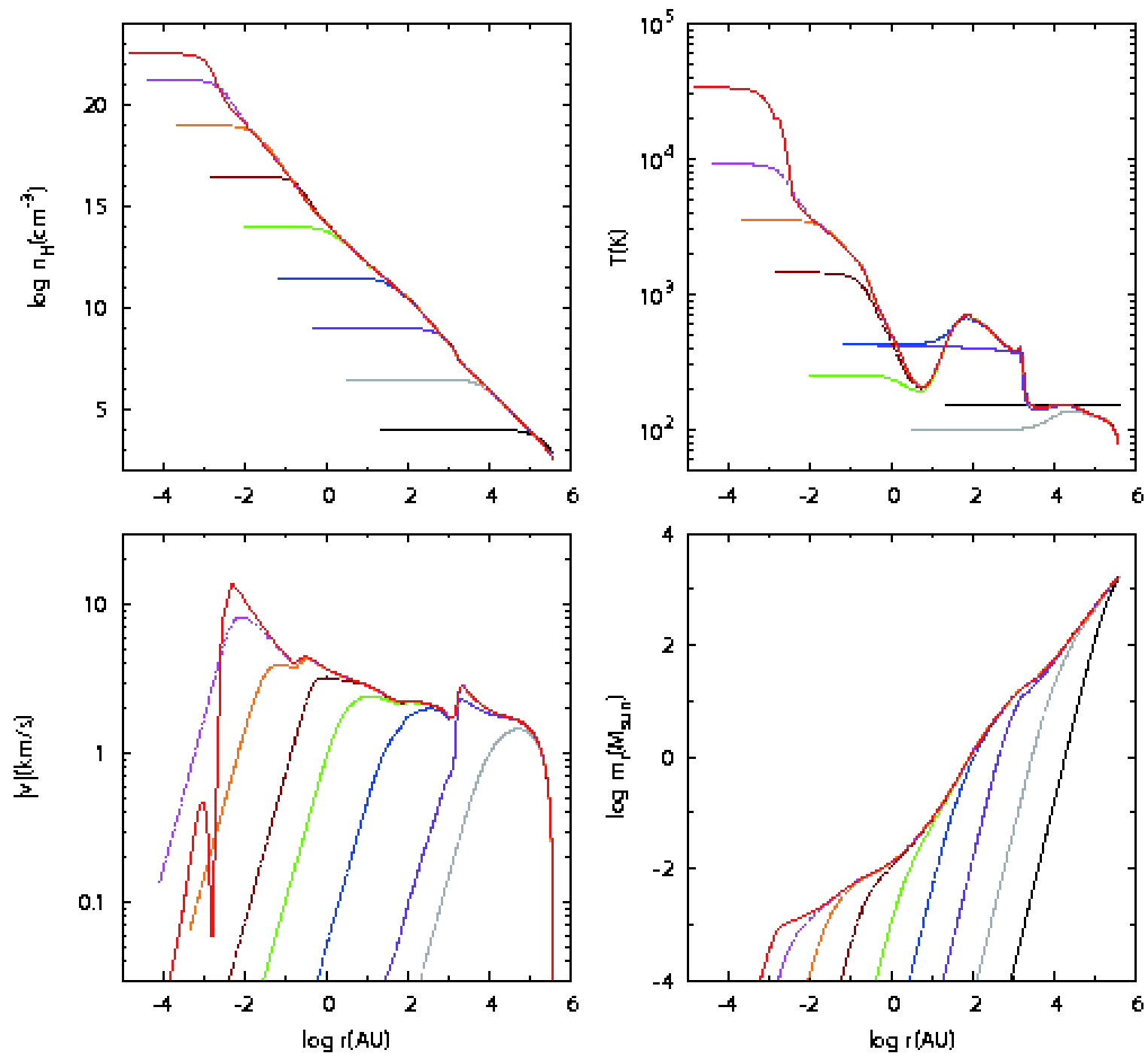

FIG. B9. - The same as Figure B7 but for metallicity $[\mathrm{M} / \mathrm{H}]=-4$ 

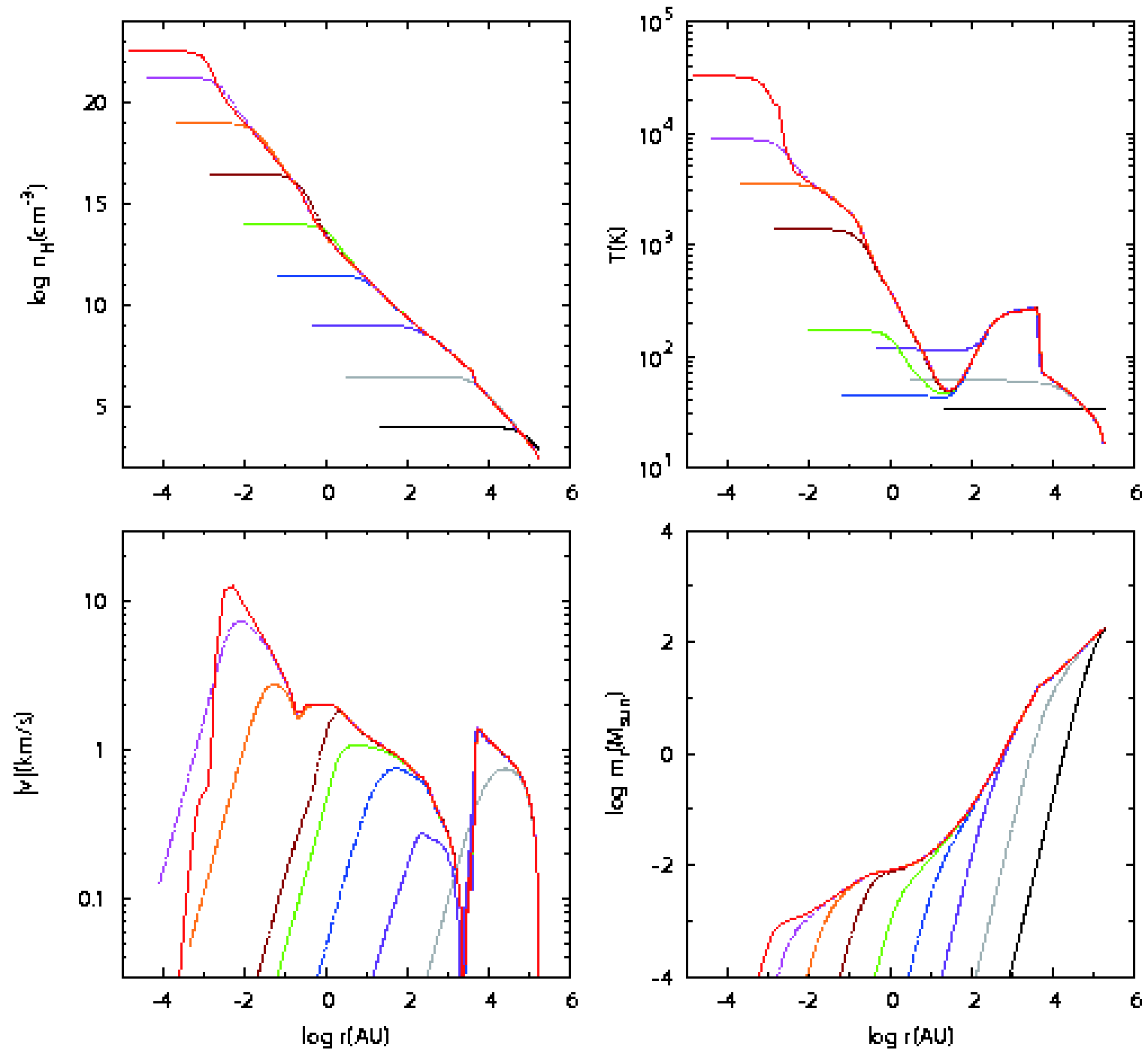

FIG. B10.- The same as Figure B7 but for metallicity $[\mathrm{M} / \mathrm{H}]=-3$ 
K. Omukai et al.
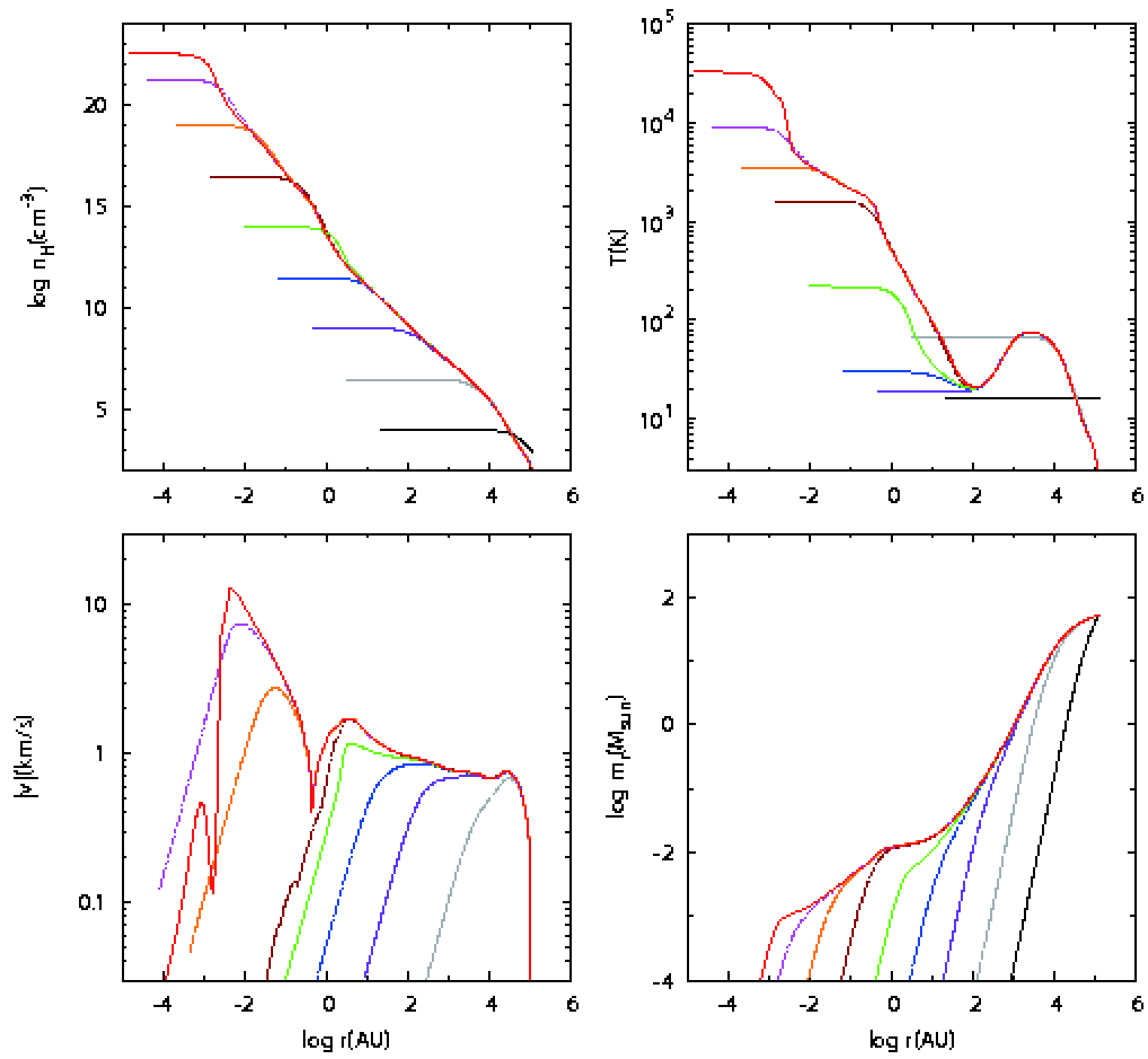

FIG. B11. - The same as Figure B7 but for metallicity $[\mathrm{M} / \mathrm{H}]=-2$ 

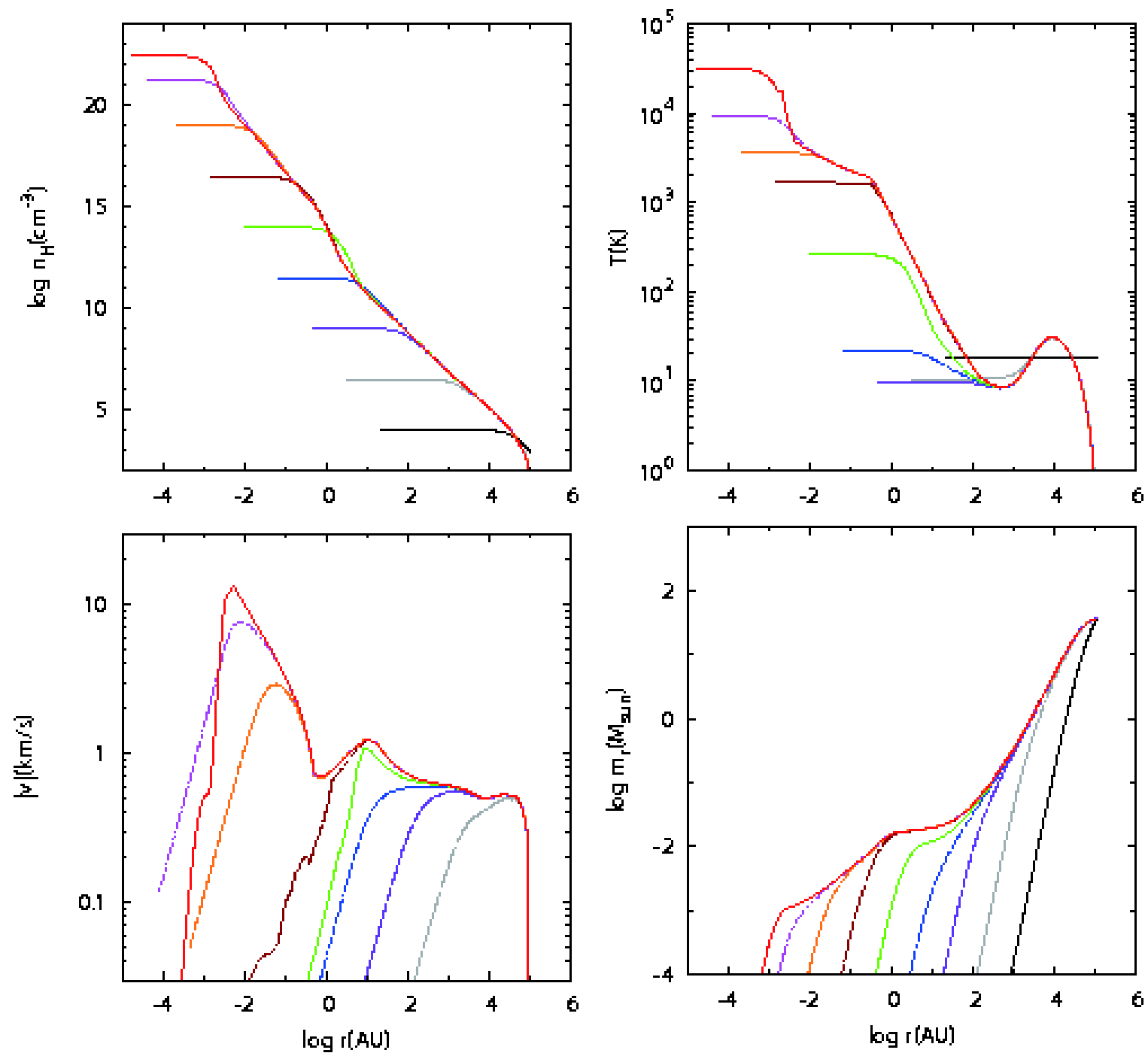

FIG. B12.- The same as Figure B7 but for metallicity $[\mathrm{M} / \mathrm{H}]=-1$ 
K. Omukai et al.
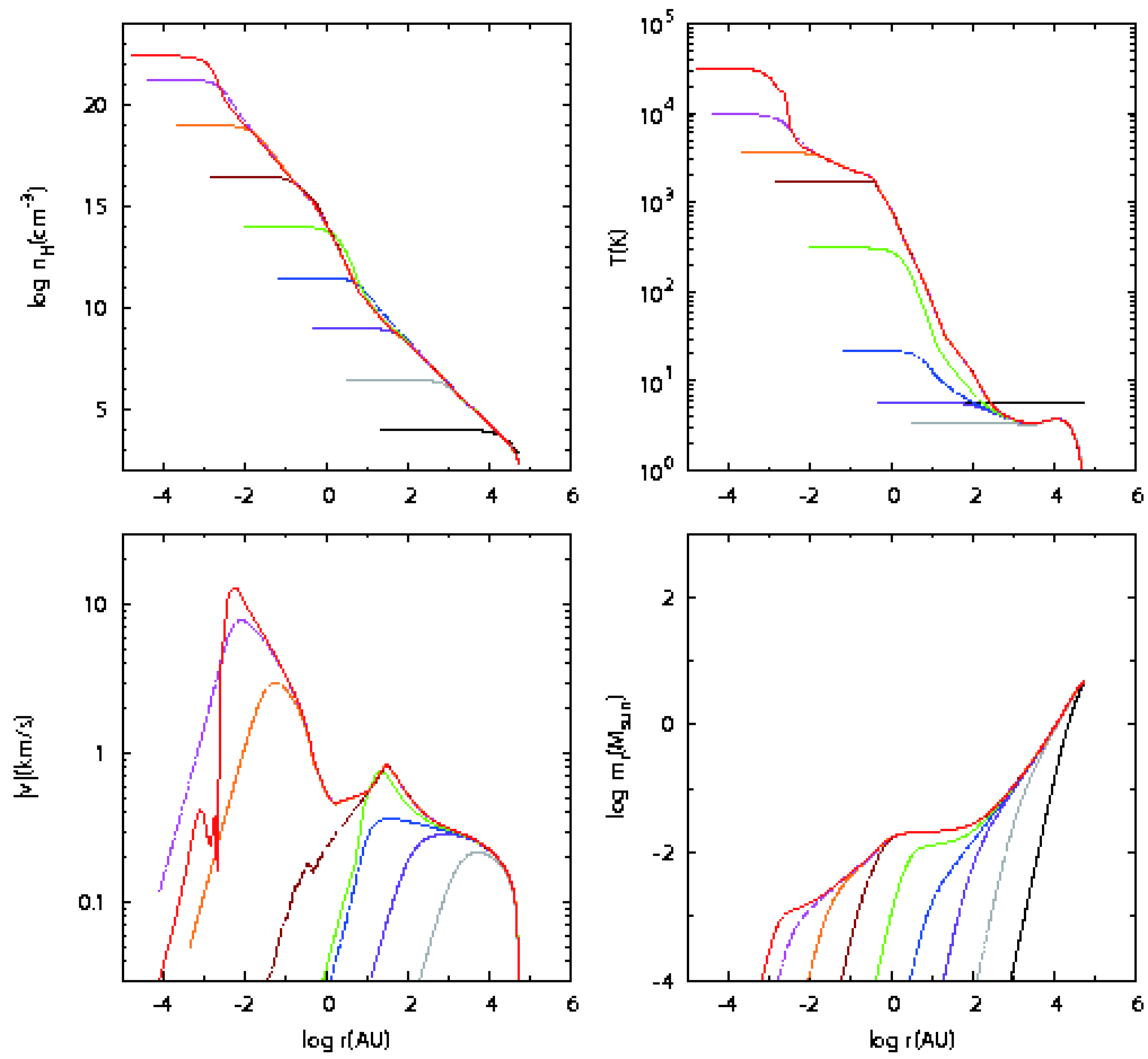

FIG. B13. - The same as Figure B7 but for metallicity $[\mathrm{M} / \mathrm{H}]=0$ 


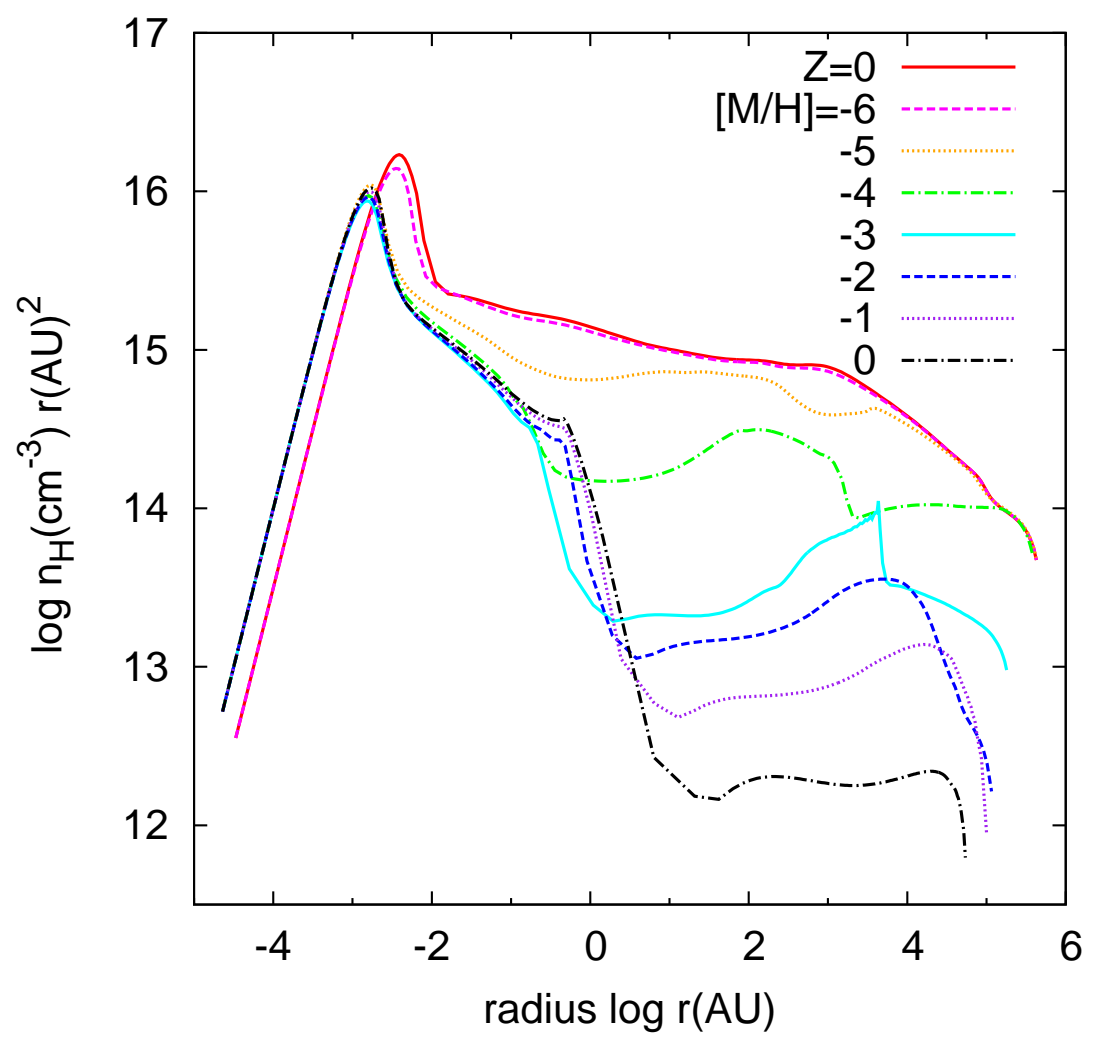

FIG. B14. - The radial distributions of density at the protostar formation epochs for the cloud cores with different metallicities. To emphasize differences among the cases, the number density times radius squared $n_{\mathrm{H}}\left(\mathrm{cm}^{-3}\right) r^{2}(\mathrm{AU})$ is shown.

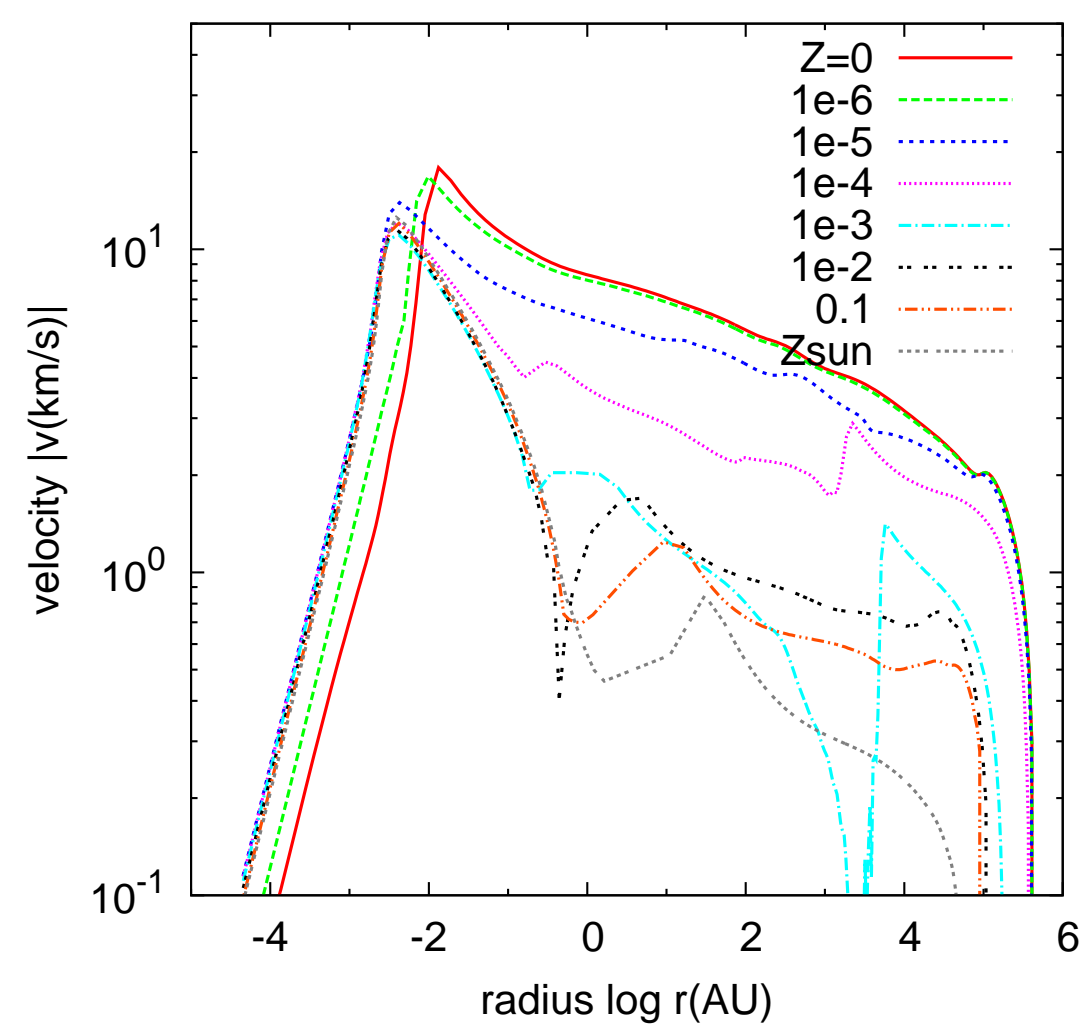

FIG. B15.- The radial distributions of velocity at the protostar-formation epochs for the cloud cores with different metallicities. 


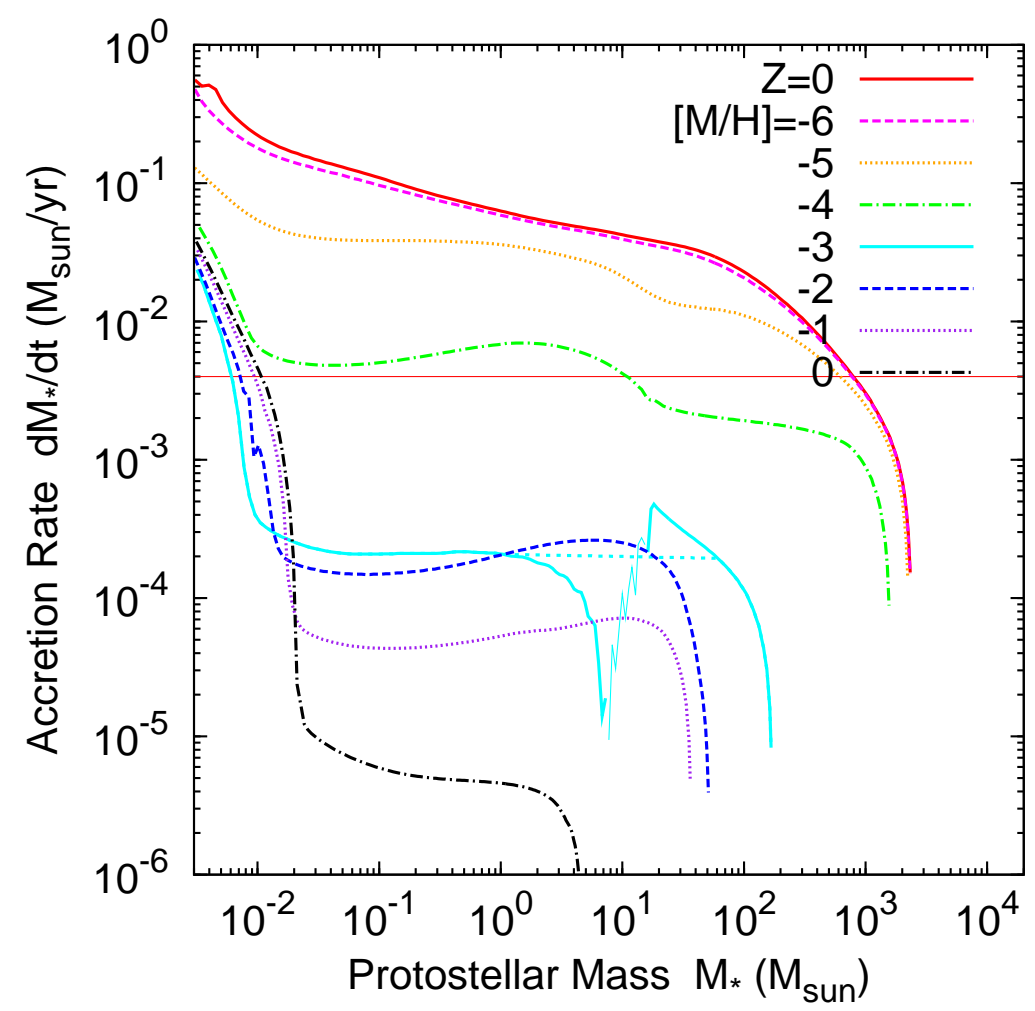

FIG. B16. - The mass accretion rates as functions of the protostellar mass derived by equation (13). The horizontal line at $d M_{*} / d t=$ $4 \times 10^{-3} M_{\odot} / \mathrm{yr}$ indicates the value above which the stationary accretion becomes impossible by the radiation force due to the accretion luminosity during the Kelvin-Helmholtz contraction. 


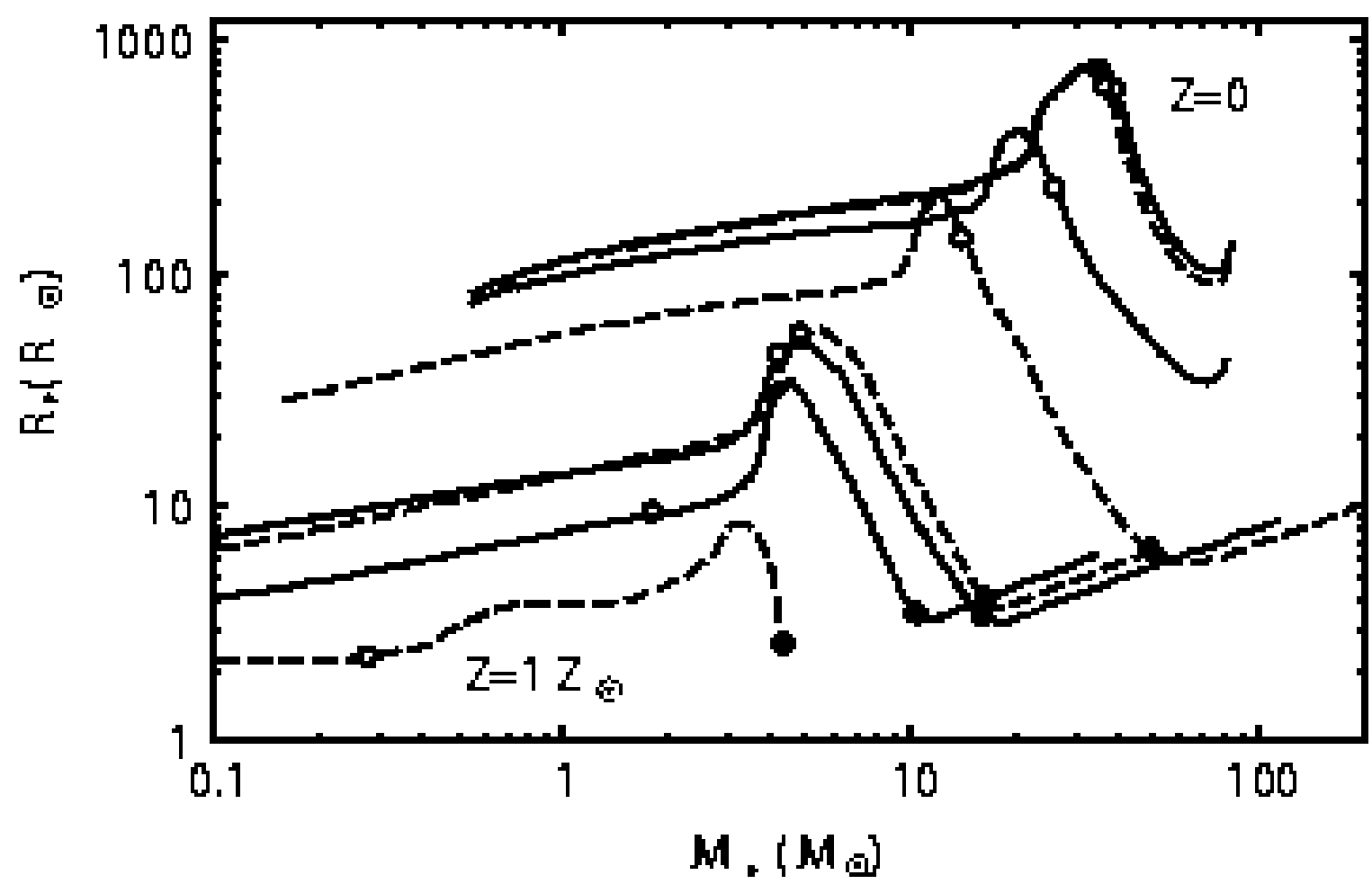

FIG. B17.- The evolution of protostellar radius during the accretion phase. The thick (dashed) lines represent the evolution with different metallicities of $Z=0,10^{-5} Z_{\odot}, 10^{-3} Z_{\odot}$, and $10^{-1} Z_{\odot}$ in descending order $\left(Z=1 Z_{\odot}, 10^{-2} Z_{\odot}, 10^{-4} Z_{\odot}\right.$, and $10^{-6} Z_{\odot}$ in ascending order). The open circles on the lines indicate the epoch when the total energy production rate by deuterium burning reaches $80 \%$ of the steady burning rate $L_{\mathrm{D}, \text { st }} \equiv \dot{M} \delta_{\mathrm{D}}$, where $\delta_{\mathrm{D}}$ is the energy available from the deuterium burning per unit gas mass. The filled circles mark the epoch when the total energy production rate by hydrogen burning exceeds $80 \%$ of the luminosity at the stellar surface $L_{*}$. 


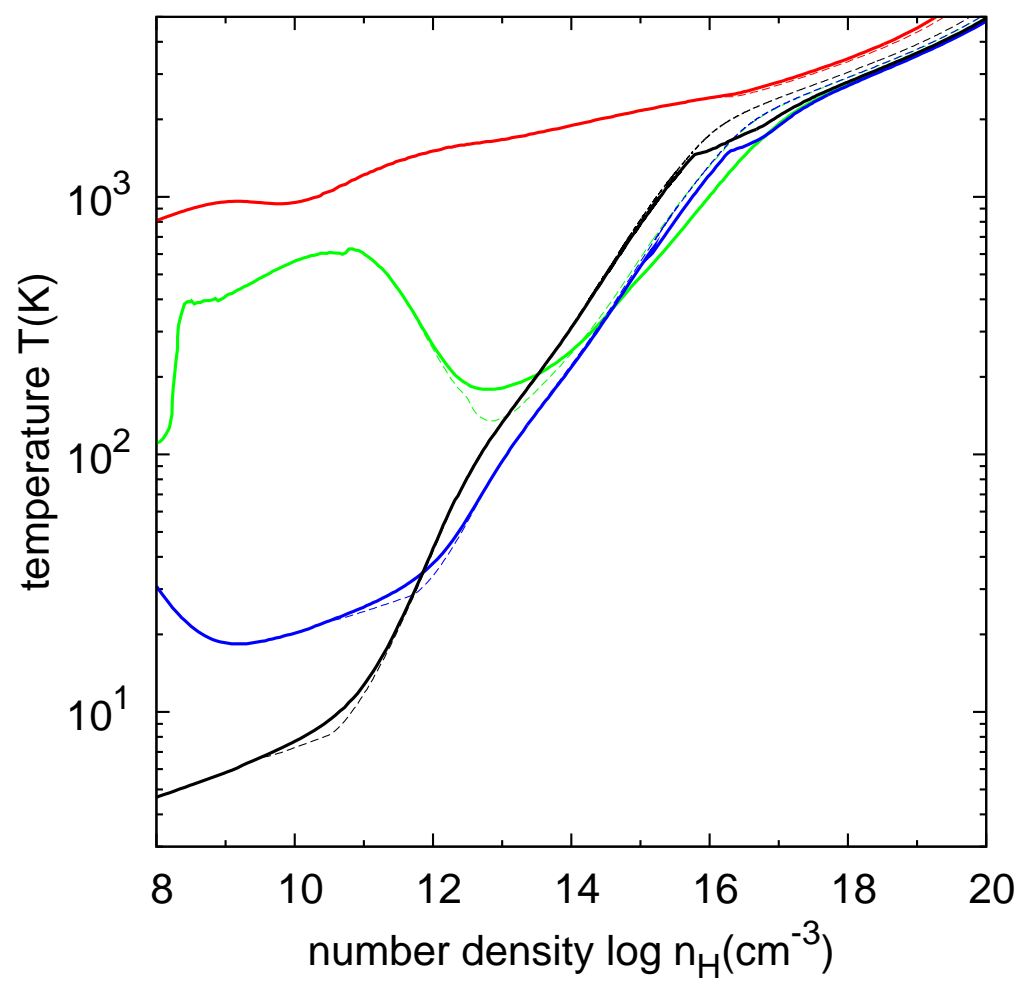

FIG. B18. - Radiative transfer effects on the central temperature evolution. The solid lines are the cases with radiative transfer (same as Fig B2 but enlarged for clarity), while dashed lines are the cases with continuum cooling rate reduced as in equation (14). Although the temperatures are slightly higher near optically thin to thick transition regime in the radiative transfer cases, their differences are small. 


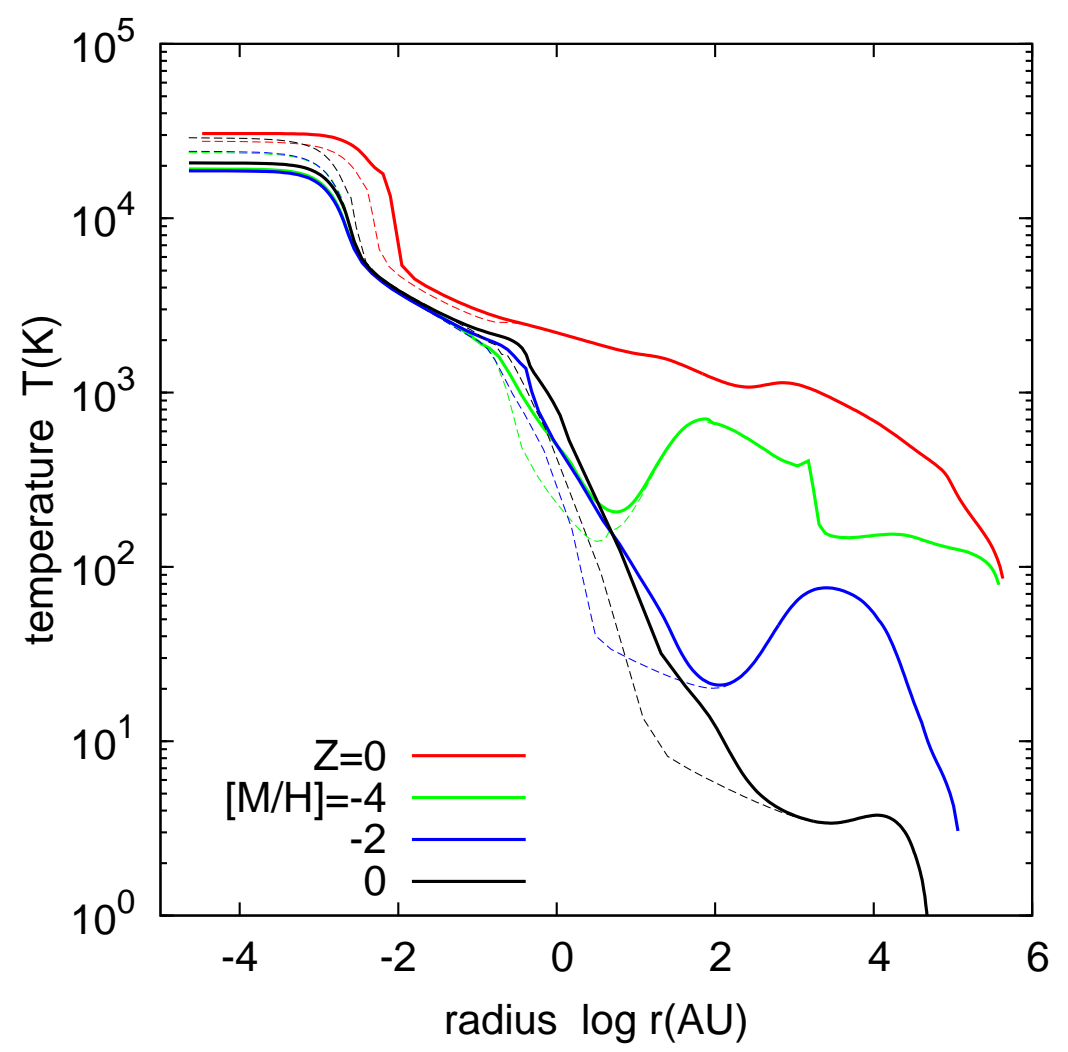

FIG. B19.- Radiative transfer effects on the envelope temperature. The solid lines are the cases with radiative transfer, while dashed lines are the cases with continuum cooling rate reduced as in equation (14). The temperatures are higher outside the first protostellar cores for the radiative transfer cases. Note that these differences are more pronouced than those in the central temperatures (Fig B18) owing to heating from inner hot regions, which is not included in the simple method by equation (14). 


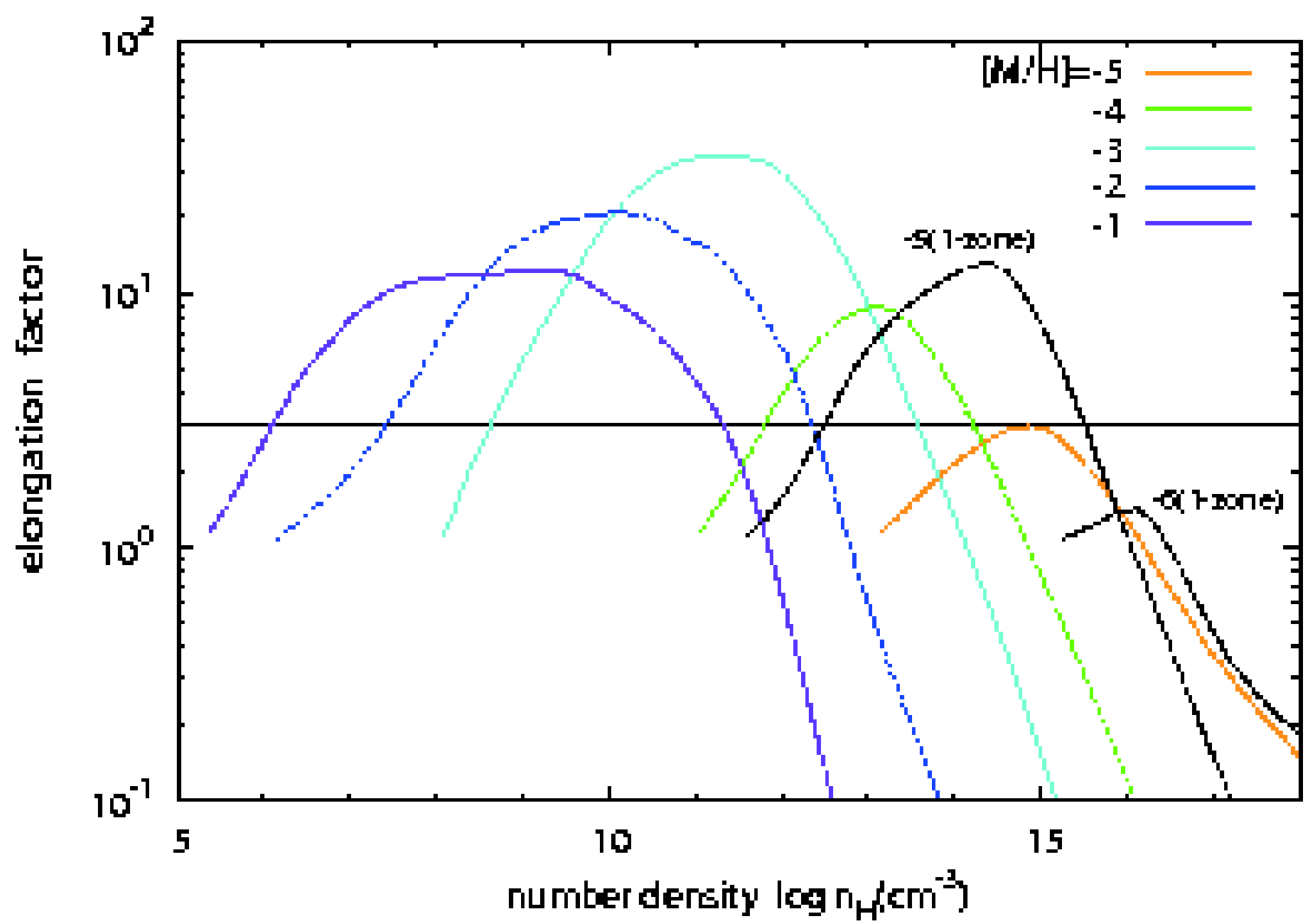

FIG. B20.- The growth and decline of elongation factors during the dust-cooling phase. The cases of metallicities $[\mathrm{M} / \mathrm{H}]=-5,-4,-3$, -2 , and -1 are shown. For comparison, the cases $[\mathrm{M} / \mathrm{H}]=-6$, and -5 by the one-zone model is also presented. The dotted horizontal line indicates the critical value $\simeq 3$ for fragmentation. 

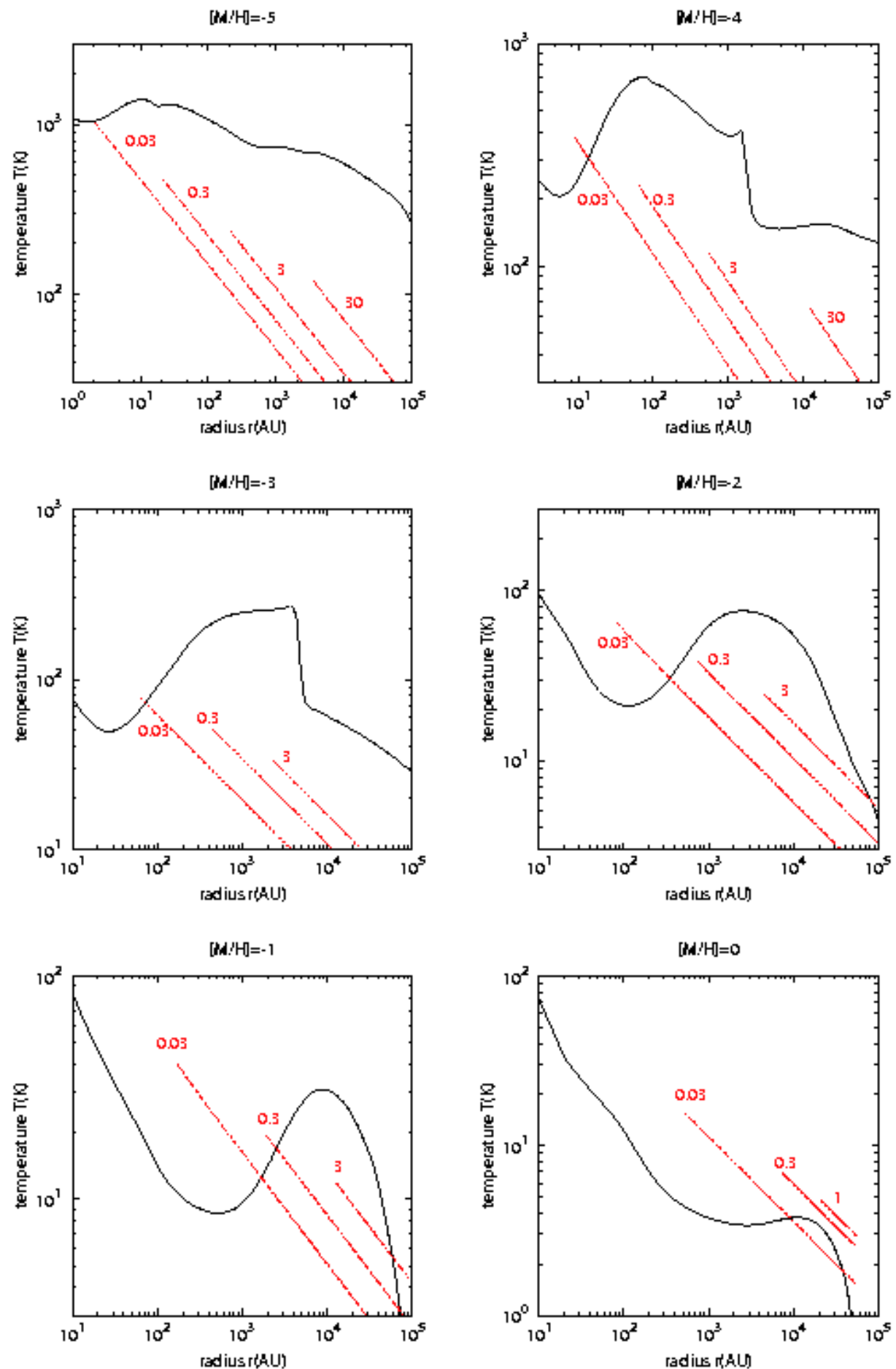

FIG. B21. - Heating effect on the ambient gas by radiation from the central protostar. The solid lines show the prestellar temperature $T_{\mathrm{pre}}$ as a function of radius. The dashed lines show the dust temperature due to protostellar radiative heating $T_{\text {heat }}=L / 16 \pi \sigma r^{2}$, where $L$ is the protostellar luminosity. The protostellar masses $M_{*}$ of those lines are indicated by numbers. The innermost point of a dashed line corresponds to the location of the rarefaction wave at $2 M_{*}$, inside of which the matter falls at almost free-fall rate and the dynamics is not affected by protostellar heating. In cases with $[\mathrm{M} / \mathrm{H}] \leq-3$, the lines for $30 M_{\odot}$ are not shown because the total core mass is less than $60 M_{\odot}$ and the entire core has been swept by the rarefaction wave at $M_{*}=30 M_{\odot}$. Similarly, the $T_{\text {heat }}$ line at $M_{*}=3 M_{\odot}$ is not illustrated in the $1 Z_{\odot}$ case, where that at $M_{*}=1 M_{\odot}$ is shown instead. In the region where $T_{\text {pre }}$ is higher than $T_{\text {heat }}$, the protostellar heating effect is negligible. Since the actual dust temperature $T_{\text {dust }}$ is determined by the balance between the cooling by its thermal radiation and heating not only by the protostellar radiation but also by the collisional coupling with gas, the dust temperature in the accretion phase roughly coincides with the higher of $T_{\text {heat }}$ or $T_{\text {pre. }}$. If $T_{\text {dust }}>T_{\text {pre }}$ (i.e., $T_{\text {heat }}>T_{\text {pre }}$ ), the gas temperature approaches $T_{\text {dust }}\left(\simeq T_{\text {heat }}\right.$ in this case) by the collisional coupling. 
TABLE B1

Reduced Chemical Reactions

\begin{tabular}{|c|c|}
\hline Number & Reaction \\
\hline 1(H1) & $\mathrm{H}^{+}+\mathrm{e} \rightarrow \mathrm{H}+\gamma$ \\
\hline $2(\mathrm{H} 2)$ & $\mathrm{H}+\mathrm{e} \rightarrow \mathrm{H}^{-}+\gamma$ \\
\hline $3(\mathrm{H} 3)$ & $\mathrm{H}^{-}+\mathrm{H} \rightarrow \mathrm{H}_{2}+\mathrm{e}$ \\
\hline $4(\mathrm{H} 4)$ & $\mathrm{H}_{2}+\mathrm{H} \rightarrow 3 \mathrm{H}$ \\
\hline $5(\mathrm{H} 5)$ & $3 \mathrm{H} \rightarrow \mathrm{H}_{2}+\mathrm{H}$ \\
\hline $6(\mathrm{H} 6)$ & $2 \mathrm{H}+\mathrm{H}_{2} \rightarrow 2 \mathrm{H}_{2}$ \\
\hline $7(\mathrm{H} 7)$ & $2 \mathrm{H}_{2} \rightarrow 2 \mathrm{H}+\mathrm{H}_{2}$ \\
\hline $8(\mathrm{H} 8)$ & $2 \mathrm{H}+$ grain $\rightarrow \mathrm{H}_{2}$ \\
\hline 11(D1) & $\mathrm{D}+\mathrm{H}^{+} \rightarrow \mathrm{D}^{+}+\mathrm{H}$ \\
\hline $12(\mathrm{D} 2)$ & $\mathrm{D}^{+}+\mathrm{H} \rightarrow \mathrm{D}+\mathrm{H}^{+}$ \\
\hline 13(D3) & $\mathrm{D}+\mathrm{H}_{2} \rightarrow \mathrm{D}+\mathrm{HD}$ \\
\hline $14(\mathrm{D} 4)$ & $\mathrm{D}^{+}+\mathrm{H}_{2} \rightarrow \mathrm{H}^{+}+\mathrm{HD}$ \\
\hline 15(D5) & $\mathrm{HD}+\mathrm{H} \rightarrow \mathrm{H}_{2}+\mathrm{D}$ \\
\hline $16(\mathrm{D} 6)$ & $\mathrm{HD}+\mathrm{H}^{+} \rightarrow \mathrm{H}_{2}+\mathrm{D}^{+}$ \\
\hline 21(M1) & $\mathrm{H}+\mathrm{CH} \rightarrow \mathrm{C}+\mathrm{H}_{2}$ \\
\hline $22(\mathrm{M} 2)$ & $\mathrm{H}+\mathrm{CH}_{2} \rightarrow \mathrm{CH}+\mathrm{H}_{2}$ \\
\hline 25(M5) & $\mathrm{H}+\mathrm{OH} \rightarrow \mathrm{H}_{2}+\mathrm{O}$ \\
\hline 26(M6) & $\mathrm{H}+\mathrm{H}_{2} \mathrm{O} \rightarrow \mathrm{OH}+\mathrm{H}_{2}$ \\
\hline $27(\mathrm{M} 7)$ & $\mathrm{H}+\mathrm{O}_{2} \rightarrow \mathrm{OH}+\mathrm{O}$ \\
\hline 28(M8) & $\mathrm{C}+\mathrm{H}_{2} \rightarrow \mathrm{CH}+\mathrm{H}$ \\
\hline 29(M9) & $\mathrm{O}+\mathrm{H}_{2} \rightarrow \mathrm{OH}+\mathrm{H}$ \\
\hline 30(M10) & $\mathrm{H}^{+}+\mathrm{O} \rightarrow \mathrm{O}^{+}+\mathrm{H}$ \\
\hline 31(M11) & $\mathrm{H}_{2}+\mathrm{CH} \rightarrow \mathrm{CH}_{2}+\mathrm{H}$ \\
\hline $34(\mathrm{M} 14)$ & $\mathrm{H}_{2}+\mathrm{OH} \rightarrow \mathrm{H}_{2} \mathrm{O}+\mathrm{H}$ \\
\hline $35(\mathrm{M} 15)$ & $2 \mathrm{OH} \rightarrow \mathrm{H}_{2} \mathrm{O}+\mathrm{O}$ \\
\hline 36(M16) & $\mathrm{OH}+\mathrm{CO} \rightarrow \mathrm{CO}_{2}+\mathrm{H}$ \\
\hline $37(\mathrm{M} 17)$ & $\mathrm{C}+\mathrm{H} \rightarrow \mathrm{CH}+\gamma$ \\
\hline 38(M18) & $\mathrm{C}+\mathrm{OH} \rightarrow \mathrm{CO}+\mathrm{H}$ \\
\hline 39(M19) & $\mathrm{C}+\mathrm{O}_{2} \rightarrow \mathrm{CO}+\mathrm{O}$ \\
\hline $40(\mathrm{M} 20)$ & $\mathrm{O}+\mathrm{H} \rightarrow \mathrm{OH}+\gamma$ \\
\hline 41(M21) & $2 \mathrm{O} \rightarrow \mathrm{O}_{2}+\gamma$ \\
\hline $42(\mathrm{M} 22)$ & $\mathrm{O}+\mathrm{CH} \rightarrow \mathrm{CO}+\mathrm{H}$ \\
\hline 44(M24) & $\mathrm{O}+\mathrm{OH} \rightarrow \mathrm{O}_{2}+\mathrm{H}$ \\
\hline $45(\mathrm{M} 25)$ & $\mathrm{H}^{+}+\mathrm{OH} \rightarrow \mathrm{OH}^{+}+\mathrm{H}$ \\
\hline 46(M26) & $\mathrm{H}^{+}+\mathrm{H}_{2} \mathrm{O} \rightarrow \mathrm{H}_{2} \mathrm{O}^{+}+\mathrm{H}$ \\
\hline $47(\mathrm{M} 27)$ & $\mathrm{H}^{+}+\mathrm{O}_{2} \rightarrow \mathrm{O}_{2}^{+}+\mathrm{H}$ \\
\hline 48(M28) & $\mathrm{C}^{+}+\mathrm{OH} \rightarrow \mathrm{CO}^{+}+\mathrm{H}$ \\
\hline 49(M29) & $\mathrm{C}^{+}+\mathrm{O}_{2} \rightarrow \mathrm{O}^{+}+\mathrm{CO}$ \\
\hline $50(\mathrm{M} 30)$ & $\mathrm{O}^{+}+\mathrm{H} \rightarrow \mathrm{H}^{+}+\mathrm{O}$ \\
\hline $51(\mathrm{M} 31)$ & $\mathrm{O}^{+}+\mathrm{H}_{2} \rightarrow \mathrm{OH}^{+}+\mathrm{H}$ \\
\hline $52(\mathrm{M} 32)$ & $\mathrm{OH}^{+}+\mathrm{H}_{2} \rightarrow \mathrm{H}_{2} \mathrm{O}^{+}+\mathrm{H}$ \\
\hline $53(\mathrm{M} 33)$ & $\mathrm{H}_{2} \mathrm{O}^{+}+\mathrm{H}_{2} \rightarrow \mathrm{H}_{3} \mathrm{O}^{+}+\mathrm{H}$ \\
\hline $54(\mathrm{M} 34)$ & $\mathrm{CO}^{+}+\mathrm{H} \rightarrow \mathrm{H}^{+}+\mathrm{CO}$ \\
\hline 55 (M35) & $\mathrm{C}^{+}+\mathrm{e} \rightarrow \mathrm{C}+\gamma$ \\
\hline 56(M36) & $\mathrm{OH}^{+}+\mathrm{e} \rightarrow \mathrm{O}+\mathrm{H}$ \\
\hline $57(\mathrm{M} 37)$ & $\mathrm{H}_{2} \mathrm{O}^{+}+\mathrm{e} \rightarrow \mathrm{OH}+\mathrm{H}$ \\
\hline 58(M38) & $\mathrm{H}_{2} \mathrm{O}^{+}+\mathrm{e} \rightarrow \mathrm{O}+\mathrm{H}_{2}$ \\
\hline 59(M39) & $\mathrm{H}_{3} \mathrm{O}^{+}+\mathrm{e} \rightarrow \mathrm{H}_{2} \mathrm{O}+\mathrm{H}$ \\
\hline $60(\mathrm{M} 40)$ & $\mathrm{H}_{3} \mathrm{O}^{+}+\mathrm{e} \rightarrow \mathrm{OH}+2 \mathrm{H}$ \\
\hline $61(\mathrm{M} 41)$ & $\mathrm{O}_{2}^{+}+\mathrm{e} \rightarrow 2 \mathrm{O}$ \\
\hline $62(\mathrm{M} 42)$ & $\mathrm{H}_{2}^{2}+\mathrm{C} \rightarrow \mathrm{CH}_{2}+\gamma$ \\
\hline
\end{tabular}


Low-Metallicity Star Formation

\begin{tabular}{|c|c|c|c|c|c|c|c|c|c|c|c|}
\hline $\log _{10}(N)$ & & & & tem & pera & ture & & & & & \\
\hline & $10 \mathrm{~K}$ & $20 \mathrm{~K}$ & $30 \mathrm{~K}$ & $50 \mathrm{~K}$ & $80 \mathrm{~K}$ & $100 \mathrm{~K}$ & $300 \mathrm{~K}$ & $600 \mathrm{~K}$ & $1000 \mathrm{~K}$ & $1500 \mathrm{~K}$ & $2000 \mathrm{~K}$ \\
\hline & & & - & $\log _{10}$ & $\left(L_{0}\right.$ & /ergs & $\mathrm{cm}^{3}$ & $\left.\mathrm{~s}^{-1}\right)$ & & & \\
\hline & 24.77 & 24.38 & 24.21 & 24.03 & 23.89 & 23.82 & 23.42 & 23.13 & 22.91 & 22.63 & 22.28 \\
\hline & & & - & $\log _{10}$ & $\left(\mathcal{L}_{\mathrm{LTE}}\right.$ & /ergs & $\mathrm{cm}^{3}$ & $\left.\mathrm{~s}^{-1}\right)$ & & & \\
\hline 14.0 & 21.08 & 20.35 & 19.94 & 19.45 & 19.01 & 18.80 & 17.81 & 17.23 & 16.86 & 16.66 & 16.55 \\
\hline 14.5 & 21.09 & 20.35 & 19.95 & 19.45 & 19.01 & 18.80 & 17.81 & 17.23 & 16.86 & 16.66 & 16.55 \\
\hline 15.0 & 21.11 & 20.37 & 19.96 & 19.46 & 19.01 & 18.80 & 17.81 & 17.23 & 16.86 & 16.66 & 16.55 \\
\hline 15.5 & 21.18 & 20.40 & 19.98 & 19.47 & 19.02 & 18.81 & 17.82 & 17.23 & 16.87 & 16.66 & 16.55 \\
\hline 16.0 & 21.37 & 20.51 & 20.05 & 19.52 & 19.05 & 18.83 & 17.82 & 17.23 & 16.87 & 16.66 & 16.55 \\
\hline 16.5 & 21.67 & 20.73 & 20.23 & 19.64 & 19.13 & 18.90 & 17.85 & 17.25 & 16.88 & 16.67 & 16.56 \\
\hline 17.0 & 22.04 & 21.05 & 20.52 & 19.87 & 19.32 & 19.06 & 17.92 & 17.28 & 16.90 & 16.69 & 16.58 \\
\hline 17.5 & 22.44 & 21.42 & 20.86 & 20.19 & 19.60 & 19.33 & 18.08 & 17.38 & 16.97 & 16.75 & 16.63 \\
\hline 18.0 & 22.87 & 21.82 & 21.24 & 20.55 & 19.95 & 19.66 & 18.34 & 17.59 & 17.15 & 16.91 & 16.78 \\
\hline 18.5 & 23.30 & 22.23 & 21.65 & 20.94 & 20.32 & 20.03 & 18.67 & 17.89 & 17.48 & 17.26 & 17.12 \\
\hline \multirow[t]{2}{*}{19.0} & 23.76 & 22.66 & 22.06 & 21.35 & 20.71 & 20.42 & 19.03 & 18.26 & 17.93 & 17.74 & 17.61 \\
\hline & & & & $\log _{10}$ & $\left(n_{1 / 2}\right.$ & $\left./ \mathrm{cm}^{-3}\right)$ & & & & & \\
\hline 14.0 & 3.29 & 3.49 & 3.67 & 3.97 & 4.30 & 4.46 & 5.17 & 5.47 & 5.53 & 5.30 & 4.70 \\
\hline 14.5 & 3.27 & 3.48 & 3.66 & 3.96 & 4.30 & 4.45 & 5.16 & 5.47 & 5.53 & 5.30 & 4.70 \\
\hline 15.0 & 3.22 & 3.45 & 3.64 & 3.94 & 4.29 & 4.45 & 5.16 & 5.47 & 5.53 & 5.30 & 4.70 \\
\hline 15.5 & 3.07 & 3.34 & 3.56 & 3.89 & 4.26 & 4.42 & 5.15 & 5.46 & 5.52 & 5.30 & 4.70 \\
\hline 16.0 & 2.72 & 3.09 & 3.35 & 3.74 & 4.16 & 4.34 & 5.13 & 5.45 & 5.51 & 5.29 & 4.68 \\
\hline 16.5 & 2.24 & 2.65 & 2.95 & 3.42 & 3.92 & 4.14 & 5.06 & 5.41 & 5.48 & 5.26 & 4.64 \\
\hline 17.0 & 1.74 & 2.15 & 2.47 & 2.95 & 3.49 & 3.74 & 4.86 & 5.30 & 5.39 & 5.17 & 4.53 \\
\hline 17.5 & 1.24 & 1.65 & 1.97 & 2.45 & 3.00 & 3.25 & 4.47 & 5.02 & 5.16 & 4.94 & 4.27 \\
\hline 18.0 & 0.742 & 1.15 & 1.47 & 1.95 & 2.50 & 2.75 & 3.98 & 4.57 & 4.73 & 4.52 & 3.84 \\
\hline 18.5 & 0.242 & 0.652 & 0.966 & 1.45 & 2.00 & 2.25 & 3.48 & 4.07 & 4.24 & 4.03 & 3.35 \\
\hline \multirow[t]{2}{*}{19.0} & -0.258 & 0.152 & 0.466 & 0.954 & 1.50 & 1.75 & 2.98 & 3.57 & 3.74 & 3.53 & 2.85 \\
\hline & & & & $\alpha$ & & & & & & & \\
\hline 14.0 & 0.439 & 0.409 & 0.392 & 0.370 & 0.361 & 0.357 & 0.385 & 0.437 & 0.428 & 0.354 & 0.322 \\
\hline 14.5 & 0.436 & 0.407 & 0.391 & 0.368 & 0.359 & 0.356 & 0.385 & 0.437 & 0.427 & 0.354 & 0.322 \\
\hline 15.0 & 0.428 & 0.401 & 0.385 & 0.364 & 0.356 & 0.352 & 0.383 & 0.436 & 0.427 & 0.352 & 0.320 \\
\hline 15.5 & 0.416 & 0.388 & 0.373 & 0.353 & 0.347 & 0.345 & 0.380 & 0.434 & 0.425 & 0.349 & 0.316 \\
\hline 16.0 & 0.416 & 0.378 & 0.360 & 0.338 & 0.332 & 0.330 & 0.371 & 0.429 & 0.421 & 0.341 & 0.307 \\
\hline 16.5 & 0.450 & 0.396 & 0.367 & 0.334 & 0.322 & 0.317 & 0.355 & 0.419 & 0.414 & 0.329 & 0.292 \\
\hline 17.0 & 0.492 & 0.435 & 0.403 & 0.362 & 0.339 & 0.329 & 0.343 & 0.406 & 0.401 & 0.317 & 0.276 \\
\hline 17.5 & 0.529 & 0.473 & 0.441 & 0.404 & 0.381 & 0.370 & 0.362 & 0.410 & 0.392 & 0.316 & 0.272 \\
\hline 18.0 & 0.555 & 0.503 & 0.473 & 0.440 & 0.423 & 0.414 & 0.418 & 0.446 & 0.404 & 0.335 & 0.289 \\
\hline 18.5 & 0.582 & 0.528 & 0.499 & 0.469 & 0.457 & 0.451 & 0.470 & 0.487 & 0.432 & 0.364 & 0.310 \\
\hline 19.0 & 0.596 & 0.546 & 0.519 & 0.492 & 0.483 & 0.479 & 0.510 & 0.516 & 0.448 & 0.372 & 0.313 \\
\hline
\end{tabular}

TABLE B2

Rotational COOLING PARAMETERS FOR CO 
K. Omukai et al.

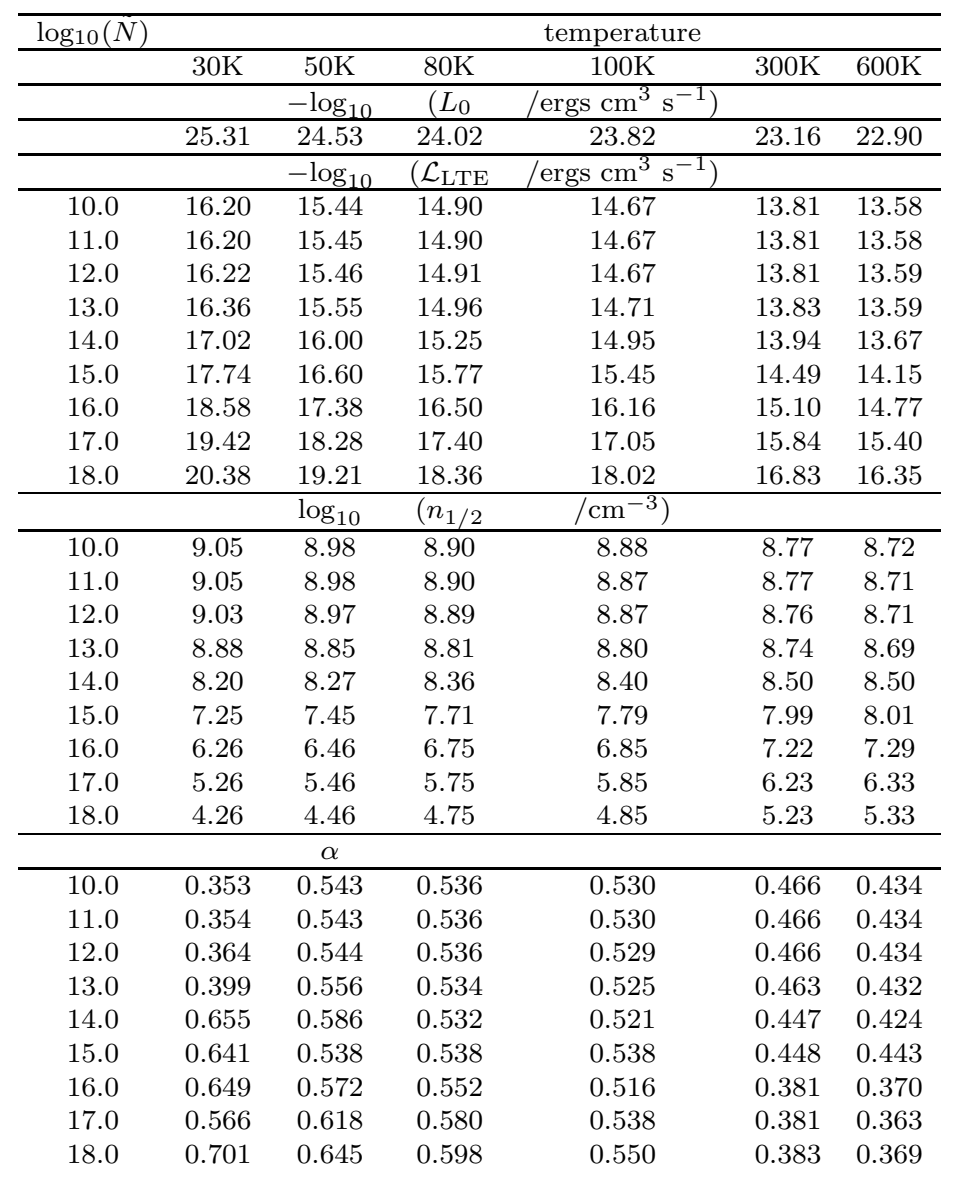

TABLE B3

ROTATIONAL COOLING PARAMETERS FOR OH 